\title{
Revelation Gap for Pricing from Samples
}

\author{
Yiding Feng * Jason D. Hartline ${ }^{\dagger} \quad$ Yingkai Li ${ }^{\ddagger}$
}

\begin{abstract}
This paper considers prior-independent mechanism design, in which a single mechanism is designed to achieve approximately optimal performance on every prior distribution from a given class. Most results in this literature focus on mechanisms with truthtelling equilibria, a.k.a., truthful mechanisms. Feng and Hartline (2018) introduce the revelation gap to quantify the loss of the restriction to truthful mechanisms. We solve a main open question left in Feng and Hartline (2018); namely, we identify a non-trivial revelation gap for revenue maximization.

Our analysis focuses on the canonical problem of selling a single item to a single agent with only access to a single sample from the agent's valuation distribution. We identify the sample-bid mechanism (a simple non-truthful mechanism) and upper-bound its prior-independent approximation ratio by 1.835 (resp. 1.296) for regular (resp. MHR) distributions. We further prove that no truthful mechanism can achieve prior-independent approximation ratio better than 1.957 (resp. 1.543) for regular (resp. MHR) distributions. Thus, a non-trivial revelation gap is shown as the sample-bid mechanism outperforms the optimal prior-independent truthful mechanism. On the hardness side, we prove that no (possibly non-truthful) mechanism can achieve prior-independent approximation ratio better than 1.073 even for uniform distributions.
\end{abstract}

\section{Introduction}

One important research direction in modern computer science focuses on multi-party computation. Two fundamental concerns in this area are (i) who should be doing what part of the computation; and (i) what are their incentives to do it correctly. The second concern has been studied extensively in the economics field of mechanism design. For the first concern, however, the system design field and the mechanism design field have different high-level guidelines. The end-to-end argument (cf. Saltzer, Reed, and Clark, 1984) - a long-standing principle in system design - suggests that the computation should be done where the data is, i.e., in a decentralized fashion. On the other hand, due to revelation principle (see next paragraph), the mechanism design literature favors systems where the entire computation is done by a center with other participants truthfully reporting their portion of the input data to the optimization. Addressing this discrepancy, in this paper, we argue that such decentralization idea from the system design field is beneficial even in purely economic terms when robust mechanisms are desired.

Revelation principle, a seminal observation in mechanism design suggests that if there is a mechanism with good equilibrium outcome, there is a mechanism which achieves the same outcome in a truthtelling equilibrium. This constructed mechanism asks agents to report true preferences, simulates the agent strategies in the original mechanism, and outputs the outcome of the simulation.

\footnotetext{
${ }^{*}$ Department of Computer Science, Northwestern University. Email: yidingfeng2021@u.northwestern.edu.

${ }^{\dagger}$ Department of Computer Science, Northwestern University. Email: hartline@northwestern.edu.

${ }^{\ddagger}$ Department of Computer Science, Northwestern University. Email: yingkai.li@u.northwestern.edu.
} 
Due to this guiding principle, a vast number of studies in mechanism design focus on truthful mechanisms (i.e., ones where revealing preferences truthfully forms an equilibria). However, successful applications - e.g., first-price auction, generalized second-price auction for advertisers in sponsored search - suggest a great practical impact for non-truthful mechanisms. From the view of multi-party computation, the mechanism itself as well as the participating agents can be thought as different parties in the system, where agents have their private preference as their input data. Truthful mechanisms correspond to systems where the optimization is done by the center (i.e. mechanism) and other parties (i.e. agents) only truthfully report their preference. Non-truthful mechanisms correspond to systems recommended by the end-to-end argument (Saltzer, Reed, and Clark, 1984), where agents are also perform some of the computation (i.e. computing their strategies).

To provide a theoretical understanding of the potential inadequacy of revelation principle and advantages of non-truthful mechanisms, we consider questions from prior-independent mechanism design, in which a mechanism is designed for agents with preferences drawn from an unknown distributions (a.k.a. prior). The goal is to identify robust mechanisms - ones with good (multiplicative) prior-independent approximation to the optimal mechanism that is tailored to the distribution of preferences. In prior-independent mechanism design, it is not generally without loss to restrict to truthful mechanisms - the equilibrium strategies for Bayesian agents in non-truthful mechanisms are a function of their prior and thus the construction of truthful mechanism via revelation principle is no longer prior-independent. Nonetheless, similar to other lines of research in mechanism design, most results in prior-independent mechanism design focus, with loss of generality, on truthful mechanisms. To understand the loss of the restriction to truthful mechanisms, Feng and Hartline (2018) introduce revelation gap, a quantification of optimal prior-independent approximation ratio among all truthful mechanisms vs. the optimal prior-independent approximation ratio among all (possibly non-truthful) mechanisms. They identify a non-trivial revelation gap for welfare-maximization. A main open question left in Feng and Hartline (2018) it to identify a non-trivial revelation gap in any canonical model for revenue maximization, which is another important and presumably technically more challenging objective in mechanism design.

\section{Main Results}

In this paper, we focus on revenue maximization in a canonical single-item environment for a single agent with a single sample access, i.e., the agent's value is drawn from an unknown distribution but the mechanism can access a single sample (independent to agent's value) from that distribution (cf. Dhangwatnotai, Roughgarden, and Yan, 2015; Allouah and Besbes, 2019). The agent knows her private valuation and the distribution for valuation, but she does not know the sample of the mechanism. Our main theorem identifies a non-trivial revelation gap for revenue maximization in this model. This theorem follows from three results. First, we introduce the (non-truthful) samplebid mechanism and obtain an upper bound of its prior-independent approximation ratio. Second, we obtain a lower bound of the optimal prior-independent approximation ratio among all possible mechanisms. Third, we show that any truthful mechanism 1 is equivalent to a sampled-based pricing mechanism introduced by Allouah and Besbes (2019) where the authors lower-bound and upper-bound the optimal prior-independent approximation ratio among all sample-based pricing mechanisms. See Table 1 for a summary of all three results. Since the prior-independent approximation ratio of the sample-bid mechanism is strictly better than the optimal prior-independent approximation ratio among all truthful mechanisms, we immediately get our non-trivial revelation

\footnotetext{
${ }^{1}$ We impose a technical assumption (i.e. scale-invariant) to the class of truthful mechanisms, which is common in prior-independent mechanism design (Allouah and Besbes, 2018, 2019; Hartline, Johnsen, and Li, 2020).
} 
Table 1: Prior-independent approximation factor of single-agent revenue-maximization with singlesample access. Two class of distributions (i.e. regular, MHR - standard assumptions in mechanism design) are considered, where MHR distributions is a subclass of regular distributions. We impose a technical assumption (i.e. scale-invariant, Definition 7.1) to the class of truthful mechanisms.

\begin{tabular}{|c|c|c|c|c|}
\cline { 2 - 5 } \multicolumn{1}{c|}{} & \multicolumn{2}{c|}{ Class of truthful mechanisms } & \multicolumn{2}{c|}{ Class of all mechanisms } \\
\cline { 2 - 5 } & Regular dists. & MHR dists. & Regular dists. & MHR dists. \\
\hline Upper bound & $1.996^{(*)}$ & $1.575^{(*)}$ & $1.835^{(\S)}$ & $1.296^{(\dagger)}$ \\
\hline Lower bound & $1.957^{(*)}$ & $1.543^{(*)}$ & \multicolumn{2}{|c|}{$1.073^{(\ddagger)}$} \\
\hline
\end{tabular}

(*) Allouah and Besbes (2019) and Lemma 7.2 .

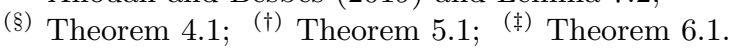

gap for revenue maximization.

In the model of a single agent with single-sample access, the class of non-truthful mechanisms is rich, which includes fairly complicated mechanisms. For example, mechanisms can ask agents to reports both her value and prior; or include multiple rounds of communication between seller and agent who sequentially reveal their private information 2 Nonetheless, our upper bound of the optimal prior-independent approximation ratio is attained by a simple non-truthful mechanism sample-bid mechanism defined as follow.

- Sample-bid mechanism: Given parameter $\alpha$ and sample $s$, the sample-bid mechanism solicits a non-negative bid $b \geq 0$, charges the agent $\alpha \cdot \min \{b, s\}$, and allocates the item to the agent if $b \geq s$.

From the agent's perspective, she reports a bid to compete for the item against a random sample realized from the same valuation distribution; and regardless of whether she wins or loses, she will always be charged $\alpha \cdot \min \{b, s\}$. In fact, the agent's optimal bidding strategy could be overbidding or underbidding, depending on the value as well as the distribution. The sample-bid mechanism has the similar format as the Becker-DeGroot-Marschak method (Becker. DeGroot, and Marschak, 1964) which has been studied and implemented in experimental economics for understanding agents' perception of the random event.

In order to beat the optimal prior-independent approximation ratio among all truthful mechanisms, we need to show the approximation for the sample-bid mechanism is strictly better than $1.957<2$ for regular distributions, and $1.543<e /(e-1)$ for MHR distributions. However, most approximation techniques and results for non-truthful mechanisms in the literature only provide similar or or larger constants - for instance, smoothness property, permeability, and revenue covering property in price of anarchy (cf. Roughgarden. Syrgkanis, and Tardos, 2017; Dütting and Kesselheim, 2015; Hartline, 2016, see more discussion in related work) 33 One the other hand, analyzing the approximation of truthful mechanisms is relatively easier. In revenue maximization, one analysis approach used extensively for truthful mechanisms is the revenue curve reduction (see next paragraph). This approach has lead to tight or nearly tight results in both prior-independent approx-

\footnotetext{
${ }^{2}$ Recall that the agent knows the distribution of the sample but does not know its realization.

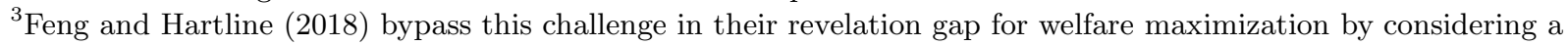
model where the all-pay auction (cf. Maskin, 2000) achieves prior-independent approximation ratio 1, i.e., it is indeed the Bayesian optimal mechanism.
} 
imation (Allouah and Besbes, 2018, 2019; Hartline, Johnsen, and Li, 2020) and Bayesian approximation (Alaei. Hartline. Niazadeh, Pountourakis, and Yuan, 2019; Jin, Lu, Tang, and Xiao, 2020; Jin, Lu, Qi, Tang, and Xiao, 2019).

Revenue curves (cf. Bulow and Roberts, 1989) give an equivalent representation of agent's valuation distribution and enable clean characterizations of the revenue of any mechanism (see e.g. Myerson, 1981; Bulow and Roberts, 1989; Alaei. Fu. Haghpanah, and Hartline, 2013). The high-level goal of revenue curve reduction is to identify a subclass of revenue curves that has closed form and over which the worst approximation guarantee is attained. The main argument is to design a (problem or mechanism) specific modification to the revenue curve (converting an arbitrary revenue curve into a revenue curve from the subclass) and analyze the impact of revenue from the modification on the given mechanism. Note that revenue is the expected payment of the agents when they bid optimally. For truthful mechanisms, after the modification has been designed, it is sufficient to study how payment changes for every bid in the modification, since agents are bidding truthfully (i.e. bids equal values). However, for non-truthful mechanisms, converting a revenue curve to another one will lead to changes in both the payment for each bid and the optimal bidding strategy of each agent. This makes the revenue curve reduction approach more difficult for non-truthful mechanisms, and thus, results of non-truthful mechanisms in the literature rarely uses this technique. In this paper, due to the simplicity of our model and the sample-bid mechanism, we are able to apply this technique by carefully (but relatively loosely) disentangling these two impacts and then analyzing them separately.

Our final result for the single-agent pricing from samples model provides a lower bound on the optimal prior-independent approximation ratio among the class of all mechanisms. This result contrasts with multi-agent models where there there exists complicated and arguably impractical non-truthful mechanism whose prior-independent approximation is arbitrarily close to 1.4 The crucial observation for proving this lower bound is that for pointmass distributions, the agent perfectly knows the seller's sample. Thus, she can strategically imitate the behavior of the values in other distributions. This restricts the seller's ability to extract revenue from the agent, which leads to a prior-independent approximation ratio at least 1.073 even on the restricted subclass of MHR distributions (in fact, even on uniform distributions). Our lower bound also suggests that it will be non-trivial to identify the non-truthful mechanism which attains the optimal prior-independent approximation ratio.

It should be noted that our better-performing non-truthful prior-independent mechanisms do not come without drawbacks relative to truthful prior-independent mechanisms. Elegantly, truthful prior-independent mechanisms do not require prior knowledge by any party. In contrast, nontruthful prior-independent mechanisms generally require some knowledge of the prior on the part of the agents. From this perspective, our results show that a seller is able to extract strictly higher revenue from the agent by taking advantage of information that the agent possesses and is able to strategize with respect to.

\section{Important Directions}

Despite the practical importance of non-truthful mechanisms, the literature on mechanism design almost exclusively considers the design of truthful mechanisms. Thus, the most general direction from this paper is to systematically build a theory for the design of non-truthful mechanisms with good

\footnotetext{
${ }^{4}$ Such mechanisms are designed and analyzed in non-parametric implementation theory - a line of research in economics, see the survey of Jackson (2001) and further discussion in the related work section.
} 
performance guarantee. Some recent works on this topic are equilibrium analysis of i.i.d. rank-based mechanism (Chawla and Hartline, 2013), robust analysis of welfare and revenue for classic mechanisms in practice (i.e. price of anarchy, see discussion in related work), estimating revenue and welfare in a mechanism from equilibrium bids in another mechanism (Chawla, Hartline, and Nekipelov, 2014b, 2016), and the sample complexity of non-truthful mechanisms in asymmetric environments (Hartline and Taggart, 2019).

Though Feng and Hartline (2018) and this paper demonstrate non-trivial revelation gap for both welfare-maximization and revenue maximization, both gaps are constant. Thus, one interesting open question left is to identify a superconstant revelation gap in a canonical model where simple non-truthful mechanisms are sufficient to beat the optimal prior-independent truthful mechanisms, and we conjecture that the single-agent with single-sample access model without any regularity assumption on distributions might be a good candidate to answer this question.

Prior-independent mechanism design for a single item with symmetric agents is an extensively studied model (Bulow and Klemperer, 1996; Devanur et al., 2015; Fu et al., 2015). The fundamental difficulty is to pin down the optimal prior-independent approximation ratio even for the two-agent setting. Recently, Allouah and Besbes (2018) obtain the tight bounds of optimal prior-independent truthful mechanism for MHR distributions, and Hartline, Johnsen, and Li (2020) generalize it to regular distributions. In both works, the main technique is the revenue curve reduction. An open question here is to identify simple non-truthful mechanism which outperforms the optimal priorindependent truthful mechanism in this canonical single-item two-agents model.

In this work, we apply the revenue curve reduction approach - a powerful technique of approximation analysis for truthful mechanisms - to a non-truthful mechanism. Our argument is not as general as ones for truthful mechanisms and thus there are gaps between the lower bound and upper bound. Besides sharpening these bounds as an open question, an important open question is to design general analysis framework on revenue curves for non-truthful mechanisms.

\section{Related Work}

Prior-independent mechanism design, as a standard framework for understanding the robustness of mechanisms, has been applied to single-dimensional mechanism design (Dhangwatnotai et al., 2015, citealpRTY-12, Fu et al., 2015, Allouah and Besbes, 2018, Feng and Hartline, 2018, Hartline et al., 2020), multi-dimensional mechanism design (Devanur et al., 2011, Roughgarden et al., 2020, Goldner and Karlin, 2016), makespan minimization (Chawla. Hartline. Malec, and Sivan, 2013), mechanism design for risk-averse agents (Fu. Hartline, and Hov, 2013), and mechanism design for agents with interdependent values (Chawla, Fu, and Karlin, 2014a). Except Fu, Hartline, and Hoy (2013) and Feng and Hartline (2018), all other results focus on truthful mechanisms.

There is a significant area of research studying mechanism design with sample access from the distribution of agents' preference, which has two regimes - small number of samples, and large number of samples. In the former regime, literature studies the approximation of mechanisms with a singlesample access (Azar. Kleinberg. and Weinberg, 2014; Dhangwatnotai. Roughgarden. and Yan, 2015; Allouah and Besbes, 2019; Feng. Hartline. and Li, 2019; Correa. Dütting. Fischer. and Schewior, 2019; Dütting, Fusco, Lazos, Leonardi, and Reiffenhäuser, 2020; Correa, Cristi, Epstein, and Soto, 2020), and mechanisms with two-sample access (Babaioff, Gonczarowski, Mansour, and Moran, 2018; Daskalakis and Zampetakis, 2020). In the latter regime, the goal is to minimize the sample complexity, i.e., number of sample to achieve $(1-\epsilon)$-approximation (e.g. Cole and Roughgarden, 2014; Morgenstern and Roughgarden, 2015; Huang et al., 2018; Gonczarowski and Weinberg, 2018; 
Guo et al., 2019; Hartline and Taggart, 2019). Except Hartline and Taggart (2019), all other results focus on truthful mechanisms.

Price of anarchy studies how classic non-truthful mechanisms (e.g. first-price auction, all-pay auction) approximate the optimal welfare. Syrgkanis and Tardos (2013) introduce a smoothness property defined on mechanisms and give an analysis framework based on this property. With this smoothness framework, the authors upper-bound the welfare-approximation of the first-price auction by $e /(e-1)$, and the welfare-approximation of the all-pay auction by 2 . These two results are later tightened by Christodoulou, Sgouritsa, and Tang (2015) for the all-pay auction and Hoy, Taggart, and Wang (2018) for the first-price auction using some mechanism-specific arguments. Hartline, Hoy, and Taggart (2014) introduce a geometric framework for analyzing the price of anarchy for both welfare and revenue. As the instantiations of the framework, authors upperbound the revenue approximation of the first-price auction with individual monopoly reserve by $2 e /(e-1)$. Dütting and Kesselheim (2015) show that bounds from these analysis frameworks are tight up to constant factors.

The literature on non-parametric implementation theory considers the same question as priorindependent mechanism design but allows mechanisms where agents cross-report their beliefs on other agents' values (e.g., Jackson, 2001). Caillaud and Robert (2005) introduce a dynamic auction for single-item multi-agent settings which is able to implement the Bayesian revenue optimal auction (Myerson, 1981) without the knowledge of agents' distribution. Dasgupta and Maskin (2000) introduce a generalization of VCG auction for multi-agent interdependent value settings 5 In this auction, agents are asked to submit a function that gives a bid for every possible valuation of the other agents. Though this auction requires no knowledge of agents' distributions, Dasgupta and Maskin (2000) show that it is Bayesian welfare-optimal under mild assumptions. Azar et al. (2012) study how to use scoring rules to learn agents' distribution and implement the auction based on this learned distribution. All results above suggest that in the multi-agent settings, there exist complicated and arguably impractical non-truthful mechanisms whose priorindependent approximation equal or are arbitrarily close to 1 . However, as we mentioned earlier, in the model of a single-agent with single-sample access, we provide a lower bound on the optimal prior-independent approximation without any restriction on mechanisms.

\section{Preliminaries}

Model. This paper focuses on the single-item revenue-maximization problem with a single agent. The agent has a private value $v$ drawn from a valuation distribution (a.k.a. prior) $F$ supported on $[\underline{v}, \bar{v}]$. we assume that distribution $F$ has positive density $f$ every where in the support. Given allocation $x$ and payment $p$, the utility of the agent is $v x-p$.

We consider the prior-independent mechanism design with a single sample access. Namely, the seller does not know the valuation distribution $F$, but has a single sample $s$ drawn from $F$. The agent knows the valuation distribution $F$ but does not observe the sample $s$, and the value $v$ of the agent is independent of the sample $s$. A mechanism $\mathcal{M}=(\tilde{x}, \tilde{p})$ includes an allocation rule $\tilde{x}: \mathbb{R} \times \mathbb{R} \rightarrow[0,1]$ mapping from the agent's bid $b$ and the sample $s$ to the allocation probability of the item; and a payment rule $\tilde{p}: \mathbb{R} \times \mathbb{R} \rightarrow \mathbb{R}_{+}$mapping from the agent's bid $b$ and the sample $s$ to the payment charged from the agent. Let $\tilde{x}(b, F)=\mathbf{E}_{s \sim F}[\tilde{x}(b, s)], \tilde{p}(b, F)=\mathbf{E}_{s \sim F}[\tilde{p}(b, s)]$ be the

\footnotetext{
${ }^{5}$ In general, there is no incentive compatible mechanism which outputs the welfare-optimal outcomes in interdependent value settings.
} 
expected allocation and payment over the randomness of the sample $s$ drawn from distribution $F$. The seller first announce the mechanism $\mathcal{M}=(\tilde{x}, \tilde{p})$ to the buyer, and then the sample $s$ and value $v$ are realized from distribution $F$. The agent report a bid $b$ based on her private value $v$, and the seller implements the mechanism $\mathcal{M}$ with input $b$ and sample $s$. We assume that the seller has full commitment power on implementing the mechanism.

Given a mechanism $(\tilde{x}, \tilde{p})$ and distribution $F$, the best response of the agent is $b(\cdot, F): \mathbb{R} \rightarrow \mathbb{R}$ which maximizes her expected utility, i.e., for every value $v, b(v, F) \in \operatorname{argmax}_{b} v \cdot \tilde{x}(b, F)-\tilde{p}(b, F) 6$ A mechanism $(\tilde{x}, \tilde{p})$ is incentive compatible $(I C)$ if reporting the agent's value truthfully is her best response, i.e., $b(v, F)=v$ for all $v$ and $F$. A mechanism $(\tilde{x}, \tilde{p})$ is individual rational (IR) if the agent's utility under her best response is non-negative, i.e, $\max _{b} v \cdot \tilde{x}(b, F)-\tilde{p}(b, F) \geq 0$ for all $v$ and $F .7$

For any mechanism $(\tilde{x}, \tilde{p})$, let $x(v, F, s)=\tilde{x}(b(v, F), s)$ be the interim allocation of value $v$ given distribution $F$ and sample $s$ when the agent follows her best response, and let $p(v, F, s)=\tilde{x}(b(v, F), s)$ be the interim payment. Moreover, denote $x(v, F)=\mathbf{E}_{s \sim F}[x(v, F, s)]$ and $p(v, F)=\mathbf{E}_{s \sim F}[p(v, F, s)]$ as the expected interim allocation and payment. We often omit $F$ in the notation if it is clear from the context.

The revenue $\operatorname{Rev}_{F}[\mathcal{M}]$ of a mechanism $\mathcal{M}=(x, p)$ on distribution $F$ is the expected payment when the agent plays her best response, i.e., $\mathbf{E}_{v \sim F}[p(v, F)]$. We evaluate mechanisms by the priorindependent approximation ratio.

Definition 2.1. The prior-independent approximation ratio of a mechanism $\mathcal{M}$ over a class of distributions DISTS is defined as

$$
\Gamma(\mathcal{M}, D I S T S) \triangleq \max _{F \in D I S T S} \frac{\operatorname{Rev}_{F}\left[\mathrm{OPT}_{F}\right]}{\operatorname{Rev}_{F}[\mathcal{M}]}
$$

where $\mathbf{R e v}_{F}\left[\mathrm{OPT}_{F}\right] \triangleq \max _{p}(1-F(p)) p$ is the optimal revenue for distribution $F$ (cf. Myerson, 1981).

Revenue Curve. For any distribution $F$, let $q(v, F)=1-F(v)$ be the quantile for the distribution, and $v(q, F)$ be the value $v$ such that $q=1-F(v)$. Here we introduce the revenue curve in quantile space (cf. Bulow and Roberts, 1989), which is a useful tool in the revenue analysis.

Definition 2.2. For any valuation distribution $F$, the revenue curve $R(q, F)$ of the agent is a mapping from any $q \in[0,1]$ to the optimal revenue from an agent with value drawn from $F$ subject to the constraint that the item is allocated with ex ante probability $q$.

In the later analysis in the paper, when $F$ is clear from the context, we omit it in the notation and only use $R(q)$ to represent the revenue curve and $q(v)$ to represent the quantile of value $v$. Let $\phi(v)=v-\frac{1-F(v)}{f(v)}$ be the virtual value of the agent.

Definition 2.3. An valuation distribution $F$ is regular if the virtual value of the agent is weakly increasing.

\footnotetext{
${ }^{6}$ When there are multiple bids maximizing the utility of the agent, we allow the agent to choose any bid maximizing her utility. The revenue guarantee we obtained in this paper holds even when the agent can break tie and choose the bid minimizing the revenue of the seller.

${ }^{7}$ Note that the utility of the agent can be negative for some realization of the sample $s$, but in expectation it must be non-negative.
} 
Theorem 2.1 (Myerson, 1981). A distribution $F$ is regular if and only if the corresponding revenue curve $R(q, F)$ is concave.

Theorem 2.2 (Myerson, 1981). For any distribution $F$ and any mechanism with interim allocation and payment rule $x(v), p(v)$, the expected revenue of the seller equals the expected virtual value of the agent plus the payment of the lowest value $\underline{v}$, i.e., $\mathbf{E}_{v \sim F}[p(v)]=\mathbf{E}_{v \sim F}[x(v) \phi(v)]+p(\underline{v})$.

Finally, we define the monopoly reserve and monopoly quantile of the agent given the revenue curve $R$.

Definition 2.4. The monopoly quantile of the agent is $q_{m}=\operatorname{argmax}_{q} R(q), 8$ and the monopoly reserve of the agent is $v_{m}=R\left(q_{m}\right) / q_{m}$.

\section{The Sample-bid Mechanism}

In this section, we introduce the main mechanism considered in this paper, the sample-bid mechanism.

Definition 3.1 (sample-bid mechanism). Given parameter $\alpha$ and sample $s$, the sample-bid mechanism solicits a non-negative bid $b \geq 0$, charges the agent $\alpha \cdot \min \{b, s\}$, and allocates the item to the agent if $b \geq s$.

In the sample-bid mechanism, the agent reports her bid without knowing the realization of the sample. From her perspective, the utility $u(v, b, F)$ for her who has value $v$, reports bid $b$, and competes with sample $s \sim F$ is

$$
u(v, b, F)=v \cdot \underbrace{F(b)}_{\operatorname{Pr}_{s \sim F}[s \leq b]}-\underbrace{\alpha b \cdot(1-F(b))}_{\text {payment when } s \geq b}-\underbrace{\alpha \int_{\underline{v}}^{\max \{b, \underline{v}\}} t d F(t)}_{\text {payment when } s \leq b}
$$

Note that reporting bid equal to zero, the utility of agent is zero. Thus, sample-bid mechanismis individually rational.

Lemma 3.1. The sample-bid mechanism is individually rational.

On the other hand, reporting bid equal to agent's value is not the best response in general. We provide a characterization of agent's optimal bid as follows.

Lemma 3.2. In the sample-bid mechanism, given any parameter $\alpha$ and distribution $F$, the optimal bid $b(v, F)$ for the agent with value $v$ satisfies the constraint that

$$
v=\alpha \cdot \frac{1-F(b(v, F))}{f(b(v, F))}
$$

or $b(v, F) \in\{0, \infty\}$. Ties are broken according to the utility of the agent.

Proof. The agent's utility from reporting bid $b$ is

$$
u(v, b, F)=v \cdot F(b)-\alpha b(1-F(b))-\alpha \int_{\underline{v}}^{\max \{b, \underline{v}\}} t d F(t)
$$

\footnotetext{
${ }^{8}$ In this paper, we break tie in favor of smaller quantile. Note that all the results are not affected by the tie breaking rule.
} 
Consider the first order condition with respect to bid $b$, if the optimal bid is obtained in the interior, we have

$$
f(b)\left(v-\alpha \cdot \frac{1-F(b)}{f(b)}\right)=0
$$

as a necessary condition for the optimality of the bid $b$. Otherwise, the optimal bid is obtained on the boundary, where $b(v, F) \in\{0, \infty\}$.

Note that there might exist multiple bids $b$ that satisfies the constraint (10) in Lemma 3.2. In that case, the agent chooses the bid which satisfies (11) and maximizes her utility. Another observation (Lemma 3.3) of the sample-bid mechanism is that the expected revenue of the seller scales linearly with the valuation distribution. Since the optimal revenue scales linearly with the valuation distribution as well, to analyze the prior-independent approximation ratio of the sample-bid mechanism, we can focus on the valuation distributions such that the optimal revenue is normalized to 1.

Lemma 3.3. Denote by $r$ the revenue of the sample-bid mechanism with any parameter $\alpha$ and any valuation distribution $F^{\dagger}$. For any $\rho>0$ and distribution $F^{\ddagger}$ such that $F^{\ddagger}$ is $F^{\dagger}$ scaled by $\rho$, i.e., $F^{\dagger}(v)=F^{\ddagger}(\rho v)$ for all $v$, the revenue of the sample-bid mechanism with parameter $\alpha$ and distribution $F^{\ddagger}$ is $\rho r$.

Proof. First we show that for any value $v$, the bid of value $v$ given distribution $F^{\dagger}$ is equivalent to the bid of value $\rho v$ given distribution $F^{\ddagger}$ scaled by $\rho$. The reason is that $F^{\dagger}(v)=F^{\ddagger}(\rho v)$ and $f^{\dagger}(v)=\rho f^{\ddagger}(\rho v)$. Therefore, by Lemma 3.2, the first order condition implies that the optimal bid satisfies $b\left(\rho v, F^{\ddagger}\right)=\rho \cdot b\left(v, F^{\dagger}\right)$. Moreover, the payment satisfies

$$
\begin{aligned}
\tilde{p}\left(\rho b, F^{\ddagger}\right) & =\alpha \rho b \cdot\left(1-F^{\ddagger}(\rho b)\right)+\alpha \int_{0}^{\rho b} t \mathrm{~d} F^{\ddagger}(t) \\
& =\rho\left(\alpha b \cdot\left(1-F^{\dagger}(b)+\alpha \int_{0}^{b} t \mathrm{~d} F^{\dagger}(t)\right)=\rho \cdot \tilde{p}\left(b, F^{\dagger}\right) .\right.
\end{aligned}
$$

By taking expectation over the valuation, the expected revenue is scaled by $\rho$ as well.

We finish this section by providing two simple monotonicity properties of the sample-bid mechanism and defer other more complicated characterizations required in our analysis to the later sections.

Lemma 3.4. In the sample-bid mechanism, given any parameter $\alpha$ and distribution $F$, the expected payment for bid $b$ is monotonically non-decreasing in $b$.

Proof. By definition, the expected payment $\tilde{p}(b, F)$ of bid $b$ over the randomness of the sample $s \sim F$ is

$$
\tilde{p}(b, F)=\alpha b \cdot(1-F(b))+\alpha \int_{\underline{v}}^{\max \{b, \underline{v}\}} t \mathrm{~d} F(t)
$$

Taking the derivative with respect to bid $b$, we have

$$
\frac{\partial \tilde{p}(b, F)}{\partial b}=\alpha(1-F(b))-\alpha b f(b)+\alpha b f(b)=\alpha(1-F(b)) \geq 0 .
$$

which finishes the proof. 
Lemma 3.5. In the sample-bid mechanism, given any parameter $\alpha$ and distribution $F$, the optimal bid $b(v, F)$ is monotonically non-decreasing in value $v$.

Proof. By Myerson (1981), the equilibrium allocation of the agent is non-decreasing in value $v$. Moreover, given the auction format, the equilibrium allocation of the agent is increasing in the bid, and thus the optimal bid $b(v, F)$ is non-decreasing in the value $v$.

\section{The Sample-bid Mechanism for MHR Distributions}

In this section, we analyze the prior-independent approximation ratio of the sample-bid mechanism over the class of MHR distributions.

Definition 4.1. A distribution $F$ is $M H R$ if the hazard rate $\frac{f(v)}{1-F(v)}$ is monotone non-decreasing in $v$.

Theorem 4.1. For the sample-bid mechanism with $\alpha=0.824$, the prior-independent approximation ratio over the class of MHR distributions is between [1.295, 1.296].

The lower bound in Theorem 4.1 is shown in the following example.

Example 4.2. For the sample-bid mechanism with $\alpha=0.824$, let $F$ be the valuation distribution such that $F(v)=1-e^{-v}$ for $v \in[0,0.43)$ and $F(v)=1$ for $v \in[0.43, \infty)$. It is easy to verify that $F$ is MHR. Moreover, the optimal revenue is 0.2797 while the expected revenue of the samplebid mechanism, which equals the expected revenue of posting a price equal to 0.824 fraction of the expected welfare, is 0.2159 . Thus, the prior-independent approximation ratio of the sample-bid mechanism with $\alpha=0.824$ is at least 1.295.

Before the proof of the upper bound in Theorem 4.1, we first introduce a characterization of the agent's optimal bid when the sample distribution $F$ is MHR; and a technical property for MHR distributions.

Lemma 4.2. In the sample-bid mechanism, given any parameter $\alpha$ and $M H R$ distribution $F$, the optimal bid $b(v, F)$ for the agent with value $v$ is

$$
b(v, F)= \begin{cases}0 & \text { if } v<\alpha \mathbf{E}_{s \sim F}[s] \\ \infty & \text { otherwise }\end{cases}
$$

Proof. By the proof of Lemma 3.2, the derivative of the utility given the bid $b$ is

$$
f(b)\left(v-\alpha \cdot \frac{1-F(b)}{f(b)}\right)
$$

where the sign of the above expression flips from negative to positive only once when the bid $b$ increases from 0 to infinity since $F$ is MHR. Thus the utility is a quasi-convex function of the bid, which implies that the maximum utility is attained at extreme points, i.e., bid 0 or $\infty$. Note that the utility for bidding 0 is always 0 , while the utility for bidding $\infty$ is $u(v, \infty, F)=v-\alpha \mathbf{E}_{s \sim F}[s]$. Hence, the agent bid $\infty$ if and only her value $v$ is at least $\alpha \mathbf{E}_{s \sim F}[s]$.

Lemma 4.3 (Allouah and Besbes, 2019). For any MHR distribution with any pair of quantile and values $\left(v_{1}, q_{1}\right),\left(v_{2}, q_{2}\right)$ such that $q_{1}=q\left(v_{1}\right) \leq q_{2}=q\left(v_{2}\right)$ and $v_{1} \geq v_{2}$. Then for any $v \geq v_{2}$, we have $q(v) \geq q_{2} \cdot e^{\frac{v-v_{2}}{v_{1}-v_{2}} \cdot \ln \left(\frac{q_{1}}{q_{2}}\right)}$. 
Lemma 4.4. The expected value for any MHR distribution with monopoly quantile $q_{m}$ is $w \geq$ $\frac{q_{m}-1}{q_{m} \cdot \ln q_{m}}$.

Proof. The expected value of the agent is

$$
\int_{0}^{\infty} q(v) \mathrm{d} v \geq \int_{0}^{\frac{1}{q_{m}}} e^{v q_{m} \cdot \ln q_{m}} \mathrm{~d} v=\frac{1}{q_{m} \cdot \ln q_{m}}\left(e^{\ln q_{m}}-e^{0}\right)=\frac{q_{m}-1}{q_{m} \cdot \ln q_{m}},
$$

where the inequality holds by applying Lemma 4.3 with $q_{1}=q_{m}, v_{1}=\frac{1}{q_{m}}$ and $q_{2}=1, v_{2}=0$.

Now, we are ready to show Theorem 4.1.

Proof of the upper bound in Theorem 4.1. Fix any MHR distribution $F$. Let $w \triangleq \mathbf{E}_{v \sim F}[v]$. Note that by Lemma 4.2, our mechanism is equivalent to posting price $\alpha w$ to the agent. Next we analyze the approximation ratio by considering the cases $\alpha w \geq v_{m}$ and $\alpha w<v_{m}$ and optimize the parameter $\alpha$ such that the approximation ratio of both cases coincide. Recall that it is without loss of generality to normalize the expected revenue of the optimal mechanism to 1 , i.e., $q_{m} \cdot v_{m}=1$.

First we consider the case when $\alpha w<v_{m}=1 / q_{m}$. By Lemma 4.4, we have $w \geq \frac{q_{m}-1}{q_{m} \cdot \ln q_{m}}$ and by combining Lemma 4.3 with $\left(v_{1}, q_{1}\right)=\left(v_{m}, q_{m}\right)$ and $\left(v_{2}, q_{2}\right)=(0,1)$, we have $q(\alpha w) \geq e^{\alpha\left(q_{m}-1\right)}$. Thus, the expected revenue in this case is

$$
\alpha w \cdot q(\alpha w) \geq \frac{\alpha\left(q_{m}-1\right)}{q_{m} \cdot \ln q_{m}} \cdot e^{\alpha\left(q_{m}-1\right)} .
$$

Then we consider the case when $\alpha w \geq v_{m}=1 / q_{m}$. In this case, combining Lemma 4.3 with $\left(v_{1}, q_{1}\right)=\left(w, q_{w}\right)$ and $\left(v_{2}, q_{2}\right)=\left(v_{m}, q_{m}\right)$, where $q_{w} \geq 1 / e$ is the quantile of the welfare (see Barlow and Marshall, 1965$)$, for any value $v \geq v_{m}$, we have $q(\alpha w) \geq q_{m} \cdot e^{\frac{\alpha w-v_{m}}{w-v_{m}} \cdot \ln \left(\frac{q_{w}}{q_{m}}\right)}$. Thus the expected revenue is

$$
\alpha w \cdot q(\alpha w) \geq \alpha w \cdot q_{m} \cdot e^{\frac{\alpha w-v_{m}}{w-v_{m}} \cdot \ln \left(\frac{q_{w}}{q_{m}}\right)} \geq \alpha w \cdot q_{m} \cdot e^{\frac{\alpha w-1 / q_{m}}{w-1 / q_{m}} \cdot \ln \left(\frac{1}{e \cdot q_{m}}\right)} .
$$

By setting $\alpha=0.824$ and numerically evaluating the above expressions for all possible values of $w$ and $q_{m}$ with respective to the given constraints, we have that the expected revenue in both cases are at least 0.7717 , which guarantees approximation ratio 1.296 .

\section{The Sample-bid Mechanism for Regular Distributions}

In this section, we analyze the prior-independent approximation of the sample-bid mechanism over the class of regular distributions.

Theorem 5.1. For the sample-bid mechanism with $\alpha=0.7$, the prior-independent approximation ratio over the class of regular distributions is between $[1.628,1.835]$.

The lower bound in Theorem 5.1 is shown in the following example.

Example 5.1. For the sample-bid mechanism with $\alpha=0.7$, let $F$ be the valuation distribution such that $F(v)=\frac{0.265}{v-0.735}$ for $v \in[1, \infty)$. It is easy to verify that $F$ is regular. Moreover, the optimal revenue is 1 while the expected revenue of the sample-bid mechanismis 0.614 . Thus, the prior-independent approximation ratio of the sample-bid mechanism with $\alpha=0.7$ is at least 1.628. 
In Section 5.1, we introduce some technical characterizations of the sample-bid mechanism which will be used in the subsequent analysis. In Sections 5.2 and 5.3, we study the prior-independent approximation ratio of the sample-bid mechanism over the class of regular distributions with monopoly quantile $q_{m} \geq 0.62$ and $q_{m} \leq 0.62$ respectively. By Lemma 3.3, without loss of generality, we restrict our attention to the class of regular valuation distributions where the optimal revenue for the distributions is exactly 1 (i.e., $v_{m} \cdot q_{m}=1$ ), and then lower-bound the expected revenue of the sample-bid mechanism with $\alpha=0.7$.

Here we sketch the high-level approach to lower-bound the expected revenue of the sample-bid mechanism in both regimes (Sections 5.2 and 5.3). Given a regular distribution $F$, we define a value threshold $v^{*}(F)$ as the smallest value whose optimal bid is at least monopoly reserve $v_{m}(F)$, i.e.,

$$
v^{*}(F) \triangleq \inf \left\{v: b(v, F) \geq v_{m}(F)\right\}
$$

Denote $q\left(v^{*}(F), F\right)$ by $q^{*}(F)$. By Lemma 3.4 and Lemma 3.5, the expected revenue $\boldsymbol{R e v}_{F}(\mathrm{SB})$ of the sample-bid mechanism SB for valuation $F$ can be lower-bounded as follows,

$$
\operatorname{Rev}_{F}(\mathrm{SB})=\int_{0}^{1} p(v(q, F), F) d q \geq p\left(v^{*}(F), F\right) \cdot q^{*}(F)+\int_{q^{*}(F)}^{1} p(v(q, F), F) d q .
$$

where $p(v, F)$ is the expected payment of the agent, with value $v$ and valuation distribution $F$, in the sample-bid mechanism. We then analyze $p\left(v^{*}(F), F\right), q^{*}(F)$, and $p(v(q, F), F)$ for $q \geq q^{*}(F)$ by providing lower bounds as the functions of $q_{m}(F)$ and other some parameters of $F 9$ Finally, by numerically evaluating the value of lower bounds for all possible possible parameters, we conclude that the expected revenue in the sample-bid mechanism for all regular distribution (with monopoly revenue 1 ) is at least 0.545 , which implies the prior-independent approximation ratio $1 / 0.545 \approx$ 1.835 of the sample-bid mechanism in Theorem 5.1. The details for discretizations and numerical evaluations can be found in Appendix A. Note that the bounds for the approximation ratio of the sample-based pricing mechanisms in Allouah and Besbes (2019) are also obtained by numerical analysis, which requires solving a relatively more complicated dynamic program. In contrast, our numerical analysis only requires brute force enumeration of a few parameters.

As we discussed in Section 2, every valuation distribution $F$ can be represented by its induced revenue curve $R$ where $R(q) \triangleq q F^{-1}(1-q)$ for all $q \in[0,1]$. In the remaining of the section, all statements, notations and analysis (except Lemma 5.3) will be presented in the language of revenue curves instead of valuation distributions.

\subsection{Technical Properties of the Sample-bid Mechanism}

In this subsection, we introduce some technical characterizations of the sample-bid mechanism which will be used in the later analysis.

To establish a lower bound on the expected revenue of of a truthful mechanism, a classic approach - revenue curve reduction - (e.g. Alaei. Hartline, Niazadeh, Pountourakis, and Yuan, 2019; Allouah and Besbes, 2018) is as follows: (i) start with an arbitrary revenue curve $R_{1}$, (ii) convert it to another revenue $R_{2}$ with closed-form formula while the optimal revenue remains the

\footnotetext{
${ }^{9}$ Let $R$ be the revenue curve induced by valuation distribution $F$. In Section 5.2 we lower-bound the expected revenue as a function of $q_{m}(F)$ and $R(0)$. In Section 5.2 we lower-bound the expected revenue as a function of $q_{m}(F), q\left(v_{m}(F) / 0.7, F\right)$ and $w \triangleq \int_{q(v / 0.7, F)}^{q_{m}(F)} \frac{R(q)}{q} d q$.
} 
same, (iii) argue that the expected revenue for $R_{2}$ is at most the expected revenue for $R_{1}$ while the optimal revenue remains the same, and finally (iv) evaluate the expected revenue for $R_{2}$ for all possible parameters. In this section, we want to apply a similar approach to the sample-bid mechanism because it is a non-truthful mechanism. A new technical difficulty arises in step (iii). When comparing $R_{1}$ and $R_{2}$, for truthful mechanisms, it is sufficient to study the change in the expected payment (i.e. $\tilde{p}\left(b, R_{1}\right)$ and $\tilde{p}\left(b, R_{2}\right)$ ) for each bid $b$. However, for non-truthful mechanisms (e.g. sample-bid mechanism), the optimal bid of the agent changes when the revenue curve $R_{1}$ is replaced by $R_{2}$. In Lemma 5.2, we provide a characterization of optimal bid when we switch from $R_{1}$ to $R_{2}$ in a specific way (illustrated in Figure 1). We use it as a building block repeatedly in Section 5.2 and Section 5.3. Intuitively, the following lemma characterizes the phenomenon that increasing the revenue curve for high values does not affect the agent's preference for low bids.

Lemma 5.2. In the sample-bid mechanism, consider any quantile $q^{\dagger} \in[0,1]$ and any pair of revenue curves $R_{1}, R_{2}$ such that $R_{1}(q) \leq R_{2}(q)$ for any quantile $q \leq q^{\dagger}$ and $R_{1}\left(q^{\dagger}\right)=R_{2}\left(q^{\dagger}\right)$. Letting $b^{\dagger}=R_{1}\left(q^{\dagger}\right) / q^{\dagger}$. For any value $v$ and any bid $b^{\ddagger} \geq b^{\dagger}$, if an agent with value $v$ and revenue curve $R_{1}$ prefers bid $b^{\dagger}$ than $b^{\ddagger}$, i.e., $u\left(v, b^{\dagger}, R_{1}\right) \geq u\left(v, b^{\ddagger}, R_{1}\right)$, then an agent with value $v$ and revenue curve $R_{2}$ also prefers bid $b^{\dagger}$ than $b^{\ddagger}$, i.e., $u\left(v, b^{\dagger}, R_{2}\right) \geq u\left(v, b^{\ddagger}, R_{2}\right)$.

Proof. By the construction of our mechanism, the utility of an agent who has value $v$, revenue curve $R$ and bids $b$ is

$$
u(v, b, R)=v \cdot(1-q(b, R))-\tilde{p}(b, R)
$$

and

$$
\tilde{p}(b, v)=\alpha b \cdot q(b, R)+\alpha \int_{q(b, R)}^{1} \frac{R(q)}{q} d q .
$$

By the assumption that $R_{1}(q) \leq R_{2}(q)$ for any quantile $q \leq q^{\dagger}$ and $b^{\ddagger} \geq b^{\dagger}$, we have $q\left(b^{\ddagger}, R_{1}\right) \leq$ $q\left(b^{\ddagger}, R_{2}\right) \leq q^{\dagger}$. See Figure 1 for a graphical illustration. Thus,

$$
\begin{aligned}
& \tilde{p}\left(b^{\ddagger}, R_{1}\right)-\tilde{p}\left(b^{\dagger}, R_{1}\right)=\alpha \cdot\left(-b^{\dagger} \cdot q^{\dagger}+\int_{0}^{q^{\dagger}} \min \left\{\frac{R_{1}(q)}{q}, b^{\ddagger}\right\} d q\right) \\
\leq & \alpha \cdot\left(-b^{\dagger} \cdot q^{\dagger}+\int_{0}^{q^{\dagger}} \min \left\{\frac{R_{2}(q)}{q}, b^{\ddagger}\right\} d q\right)=\tilde{p}\left(b^{\ddagger}, R_{2}\right)-\tilde{p}\left(b^{\dagger}, R_{2}\right) .
\end{aligned}
$$

Thus,

$$
\begin{array}{r} 
\\
u\left(b^{\dagger}, v, R_{1}\right)-u\left(b^{\ddagger}, v, R_{1}\right)=v \cdot\left(1-q^{\dagger}\right)-\tilde{p}\left(b^{\dagger}, R_{1}\right)-v \cdot\left(1-q\left(b^{\ddagger}, R_{1}\right)\right)+\tilde{p}\left(b^{\ddagger}, R_{1}\right) \\
\leq v \cdot\left(1-q^{\dagger}\right)-\tilde{p}\left(b^{\dagger}, R_{2}\right)-v \cdot\left(1-q\left(b^{\ddagger}, R_{2}\right)\right)+\tilde{p}\left(b^{\ddagger}, R_{2}\right)=u\left(b^{\dagger}, v, R_{2}\right)-u\left(b^{\ddagger}, v, R_{2}\right)
\end{array}
$$

and hence $u\left(b^{\dagger}, v, R_{1}\right) \geq u\left(b^{\ddagger}, v, R_{1}\right)$ implies $u\left(b^{\dagger}, v, R_{2}\right) \geq u\left(b^{\ddagger}, v, R_{2}\right)$.

Lemma 5.3. In the sample-bid mechanism with any parameter $\alpha \in[0,1]$, for an agent with concave revenue curve $R$ and value $v$ greater than the monopoly reserve $v_{m}$, she weakly prefers the bid $v / \alpha$ than any bid $b^{\dagger} \in\left[v_{m}, v / \alpha\right]$, i.e., $u(v, v / \alpha, R) \geq u\left(v, b^{\dagger}, R\right)$. 


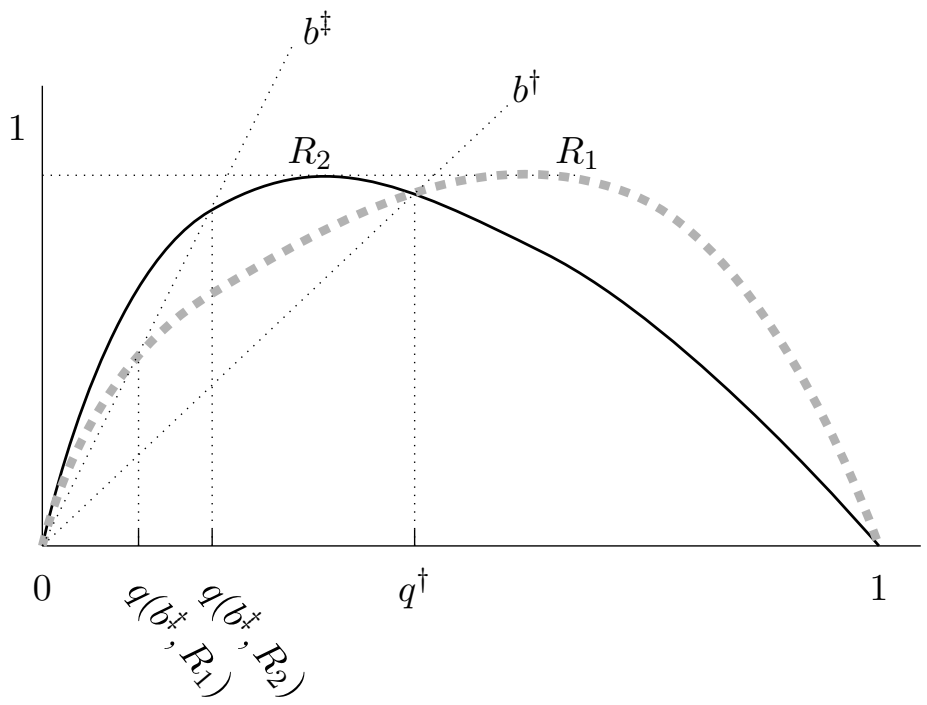

Figure 1: Graphical illustration for Lemma 5.2. The gray dashed thick (resp. black solid) curve is revenue curve $R_{1}$ (resp. $R_{2}$ ). The slopes of two dotted lines from $(0,0)$ are $b^{\ddagger}$ and $b^{\dagger}$ respectively.

Proof. Let $F$ be a regular distribution. By the definition, the utility of the agent who has value $v$, valuation distribution $F$ and bids $b$ is

$$
u(v, b, F)=v \cdot F(b)-\tilde{p}(b, F)
$$

By considering the first order condition as in Lemma 3.2, we have

$$
\frac{\partial u(v, b, F)}{\partial b}=f(b)\left(v-\alpha \cdot \frac{1-F(b)}{f(b)}\right) .
$$

Thus, we can compute the difference between $u(v, v / \alpha, F)$ and $u(v, b, F)$ for any value $v \geq v_{m}$ and bid $b \in\left[v_{m}, v / \alpha\right]$ as follows,

$$
\begin{aligned}
u(v, v / \alpha, F)-u(v, b, F) & =\int_{b}^{\frac{v}{\alpha}} \alpha f(t)\left(\frac{v}{\alpha}-\frac{1-F(t)}{f(t)}\right) d t \\
& \geq \int_{b}^{\frac{v}{\alpha}} \alpha f(t)\left(t-\frac{1-F(t)}{f(t)}\right) d t \\
& \geq 0
\end{aligned}
$$

where the last inequality uses the fact that $t-\frac{1-F(t)}{f(t)} \geq 0$ for all $t \geq v_{m}$ if $F$ is regular.

\subsection{Regular Distributions with Monopoly Quantile $q_{m} \geq 0.62$}

In this subsection, we analyze the approximation ratio of the sample-bid mechanism over the class of regular distributions with monopoly quantile $q_{m} \geq 0.62$.

Lemma 5.4. For the sample-bid mechanism with $\alpha=0.7$, the approximation ratio over the class of regular distributions with monopoly quantile $q_{m} \geq 0.62$ is at most 1.835 . 
Fix an arbitrary revenue curve $R$, let

$$
v^{*}(R) \triangleq \inf \left\{v: b(v, R) \geq v_{m}(R)\right\}
$$

be the smallest value whose optimal bid $b(v, R)$ for revenue curve $R$ is at least the monopoly reserve $v_{m}(R)$. Since Lemma 3.5 guarantees that $b(v, R)$ is weakly non-decreasing in $v, v^{*}(R)$ is well-defined, $b(v, R) \geq v_{m}(R)$ for all $v \geq v^{*}(R)$, and $b(v, R)<v_{m}(R)$ for all $v<v^{*}(R)$. Denote $q\left(v^{*}(R), R\right)$ by $q^{*}(R)$. We decompose the proof of Lemma 5.4 by considering the following two subregimes - Lemma 5.5 for revenue curve $R$ with $v^{*}(R) \leq v_{m}(R)$; and Lemma 5.7 for revenue curve $R$ with $v^{*}(R) \geq v_{m}(R)$.

Lemma 5.5. Given any concave revenue curve $R$ such that $q_{m}(R) \geq 0.62$ and $v^{*}(R) \leq v_{m}(R)$, the revenue of the sample-bid mechanism with $\alpha=0.7$ is a 1.835-approximation of the optimal revenue.

Proof. Fix an arbitrary concave revenue curve $R$ satisfying the requirement in the lemma statement, i.e., $q_{m}(R) \geq 0.62$ and $v^{*}(R) \leq v_{m}(R)$. Consider an arbitrary value $v \geq v^{*}(R)$. By Lemma 3.5, the optimal bid of an agent with value $v$ is at least $v_{m}(R)$. Thus, together with Lemma 3.4, her expected payment in sample-bid mechanism is at least the expected payment $\tilde{p}\left(v_{m}(R), R\right)$ of bidding $v_{m}(R)$, and

$$
\begin{aligned}
\tilde{p}\left(v_{m}(R), R\right) & =0.7 v_{m}(R) q_{m}(R)+0.7 \int_{q_{m}(R)}^{1} \frac{R(q)}{q} d q=0.7+0.7 \int_{q_{m}(R)}^{1} \frac{R(q)}{q} d q \\
& \geq 0.7+0.7 \int_{q_{m}(R)}^{1} \frac{\frac{1-q}{1-q_{m}(R)}}{q} d q=-\frac{0.7 \log \left(q_{m}(R)\right)}{1-q_{m}(R)} .
\end{aligned}
$$

where the inequality uses the fact that (1) $R$ is concave, which implies that $R(q) \geq \frac{1-q}{1-q_{m}(R)}$ for all $q \geq q_{m}(R)$; and $(2) v_{m}(R) q_{m}(R)$ is normalized to 1 for the revenue curve $R$. Since $v^{*}(R) \leq v_{m}(R)$, each value with quantile smaller than $q_{m}(R)$ has $\tilde{p}\left(v_{m}(R), R\right)$ as a lower bound of its payment in the sample-bid mechanism. Thus, a lower bound of the expected revenue $\operatorname{Rev}_{R}(\mathrm{SB})$ for revenue curve $R$ in the sample-bid mechanism is

$$
\begin{aligned}
\operatorname{Rev}_{R}(\mathrm{SB}) & =\int_{0}^{1} p(v(q, F), F) d q \geq p\left(v^{*}(R), R\right) \cdot q^{*}(R) \\
& \geq \tilde{p}\left(v_{m}(R), R\right) \cdot q_{m}(R) \geq-\frac{0.7 \log \left(q_{m}(R)\right) q_{m}(R)}{1-q_{m}(R)}
\end{aligned}
$$

which is at least 0.545 for all $q_{m}(R) \geq 0.62$. This finishes the proof, since we (without loss of generality) consider revenue curve $R$ with optimal revenue equal to 1 , i.e., $v_{m}(R) \cdot q_{m}(R)=1$.

Before diving into the subregime where $v^{*}(R) \geq v_{m}(R)$, we provide a characterization (Lemma 5.6) of the optimal bid for concave revenue curves with monopoly quantile greater than 0.62 . Specifically, Lemma 5.6 guarantees that $b(v, R)=0$ for all value $v<v^{*}(R)$.

Lemma 5.6. In the sample-bid mechanism with parameter $\alpha=0.7$, given any value $v$ and any concave revenue curve $R$ with $q_{m}(R) \geq 0.62$, the optimal bid $b(v, R)$ for an agent with value $v$ and revenue curve $R$ satisfies $b(v, R) \in\{0\} \cup\left[v_{m}(R), \infty\right)$.

Proof. We prove the lemma by contradiction. See Figure 2 for a graphical description of the following construction. Suppose there exists an agent who has value $v$, revenue curve $R_{1}$ s.t. 


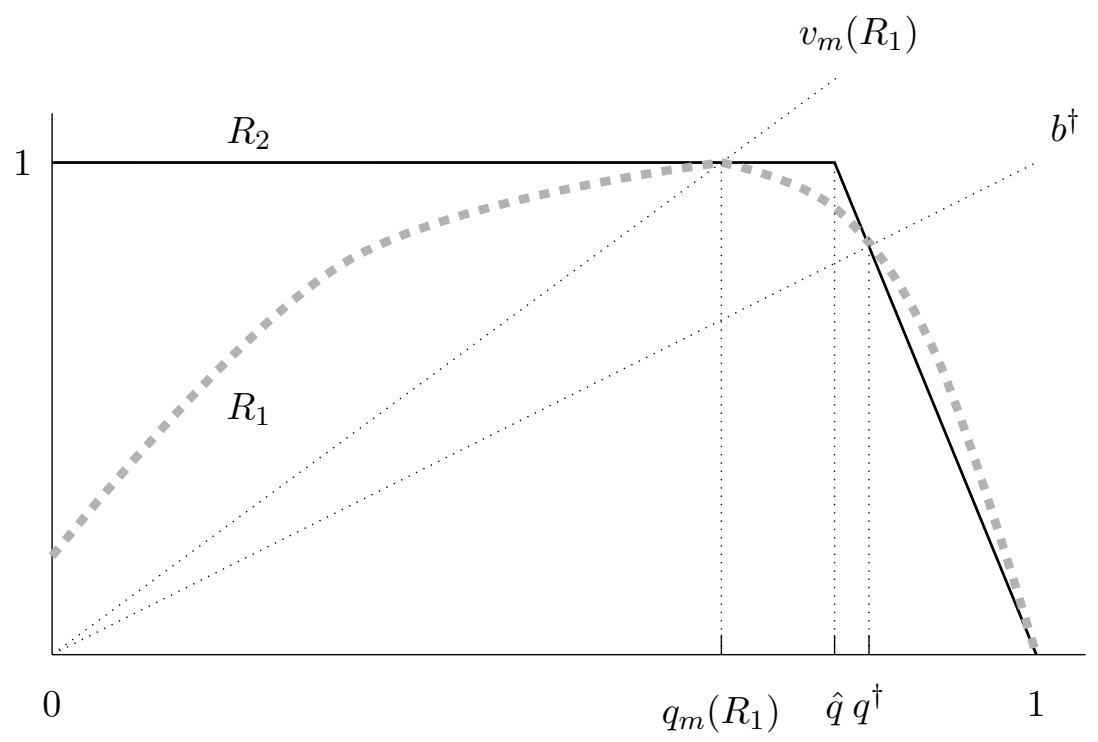

Figure 2: Graphical illustration for Lemma 5.6. The gray dashed (resp. black solid) curve is revenue curve $R_{1}$ (resp. $R_{2}$ ). The slopes of two dotted lines from $(0,0)$ are $v_{m}\left(R_{1}\right)$ and $b^{\dagger}$ respectively.

$q_{m}\left(R_{1}\right) \geq 0.62$ and strictly prefers a bid of $b^{\dagger} \in\left(0, v_{m}\left(R_{1}\right)\right)$ over all other bids. Denote $q\left(b^{\dagger}, R_{1}\right)$ by $q^{\dagger}$. Let $\hat{q} \triangleq 1-\frac{1-q^{\dagger}}{R_{1}\left(q^{\dagger}\right)}$. Now consider another revenue curve $R_{2}$ defined as follows,

$$
R_{2}(q) \triangleq \begin{cases}1 & q \in[0, \hat{q}] \\ \frac{1-q}{1-\hat{q}} & q \in[\hat{q}, 1]\end{cases}
$$

By construction, $R_{2}$ is a concave revenue curve s.t. (i) $\hat{q} \geq 0.62$; (ii) $b^{\dagger} \leq 1 / \hat{q}$; (iii) $R_{1}(q) \leq R_{2}(q)$ for all $q \in\left[0, q^{\dagger}\right]$; and (iv) $R_{1}(q) \geq R_{2}(q)$ for all $q \in\left[q^{\dagger}, 1\right]$.

Applying Lemma 5.2 on $R_{1}, R_{2}, q^{\dagger}, v$ and all $b^{\ddagger} \geq b^{\dagger}$, we conclude that the optimal bid for an agent with value $v$ and revenue curve $R_{2}$ is in $\left[0, b^{\dagger}\right]$. Furthermore, note that $u\left(v, b^{\dagger}, R_{2}\right) \geq u\left(v, b^{\dagger}, R_{1}\right)>0$ where the first inequality holds by the construction of $R_{2}, 10$ and the second inequality holds by our assumption that $b^{\dagger}$ is strictly preferred for $R_{1}$. Hence, there exists an optimal bid in $\left(0, b^{\dagger}\right]$ that is strictly preferred to biding zero and weakly preferred to all other bids for $R_{2}$. Next we argue that this leads to a contradiction by considering $v \leq 1 / \hat{q}$ and $v \geq 1 / \hat{q}$ separately.

Case (i) $v \leq 1 / \hat{q}$ : Note that for any bid $b \in[0,1 / \hat{q}]$, the utility $u\left(v, b, R_{2}\right)$ has a closed-form expression as follows,

$$
u\left(v, b, R_{2}\right)=v \frac{b(1-\hat{q})}{b(1-\hat{q})+1}+0.7 \log \left(\frac{1}{b(1-\hat{q})+1}\right) .
$$

Considering the first order condition of $u\left(v, b, R_{2}\right)$ with respect to bid $b$, after basic simplification, we have

$$
b=\frac{v}{0.7}-\frac{1}{1-\hat{q}} .
$$

\footnotetext{
${ }^{10}$ The allocation of bidding $b^{\dagger}$ is the same for both revenue curves, while the payment of bidding $b^{\dagger}$ is higher for revenue curve $R_{1}$.
} 
This leads to a contradiction since for all $\hat{q} \in[0.62,1] 11$ and $v \in[0,1 / \hat{q}]$, we have $\frac{v}{0.7}-\frac{1}{1-\hat{q}}<0$, i.e., bidding 0 is weakly preferred than any bid $b \in\left(0, b^{\dagger}\right)$.

Case (ii) $v \geq 1 / \hat{q}$ : Let $b^{\ddagger} \triangleq v / 0.7$, and $q^{\ddagger} \triangleq q\left(b^{\ddagger}, R_{2}\right)=0.7 / v$. Since $v \geq 1 / \hat{q}$, the construction of $R_{2}$ guarantees that $b^{\ddagger} \cdot q^{\ddagger}=1=R_{2}\left(q^{\ddagger}\right)$. Note that the utility $u\left(v, b^{\ddagger}, R_{2}\right)$ has a closed-form expression as follows,

$$
\begin{aligned}
u\left(v, b^{\ddagger}, R_{2}\right)=v & -2 v q^{\ddagger}+0.7(1-\hat{q})+0.7 \log (\hat{q}) \\
& -0.7\left(\hat{q}-q^{\ddagger}\right)\left(1-b^{\ddagger} q^{\ddagger}\right)\left(\hat{q}-q^{\ddagger}-q^{\ddagger} \log (\hat{q})+q^{\ddagger} \log \left(q^{\ddagger}\right)\right) .
\end{aligned}
$$

This leads to a contradiction since for all $\hat{q} \in[0.62,1], v \in[1 / \hat{q}, \infty)$, and $\left(\frac{v}{0.7}-\frac{1}{1-\hat{q}}\right) \in[0,1 / \hat{q}]$, we have $u\left(v, b^{\ddagger}, R_{2}\right) \geq u\left(v, \frac{v}{0.7}-\frac{1}{1-\hat{q}}, R_{2}\right) 12$ i.e., bidding 0 or $v / 0.7$ is weakly preferred than any bid $b \in\left(0, b^{\dagger}\right)$.

Now, we provide the approximation guarantee for revenue curve $R$ with $v^{*}(R) \geq v_{m}(R)$.

Lemma 5.7. Given any concave revenue curve $R$ such that $q_{m}(R) \geq 0.62$ and $v^{*}(R) \geq v_{m}(R)$, the revenue of the sample-bid mechanism with $\alpha=0.7$ is a 1.835-approximation of the optimal revenue.

Proof. The proof is done in four major steps:

Step 1- flattening the revenue curve for all quantile $q \geq q_{m}\left(R_{1}\right)$. Fix an arbitrary revenue curve $R_{1}$ satisfying the requirements in the lemma statement, i.e., $q_{m}(R) \geq 0.62$ and $v^{*}(R) \geq v_{m}(R)$. Consider another revenue curve $R_{2}$ defined as follows (see Figure 3a for a graphical illustration)

$$
R_{2}(q) \triangleq \begin{cases}R_{1}(q) & q \in\left[0, q_{m}\left(R_{1}\right)\right] \\ 1 & q \in\left[q_{m}\left(R_{1}\right), 1\right]\end{cases}
$$

We claim that the expected revenue of the sample-bid mechanism with $\alpha=0.7$ for revenue curve $R_{2}$ is at most that of revenue curve $R_{1}$. To see this, consider the virtual surplus for both revenue curves. By our assumption that $v^{*}\left(R_{1}\right) \geq v_{m}\left(R_{1}\right)$, every quantile $q>q_{m}\left(R_{1}\right)$ has negative virtual value $R_{1}^{\prime}(q)$ in $R_{1}$, bids zero (Lemma 5.6) and gains zero virtual surplus while their virtual value $R_{2}^{\prime}(q)$ becomes zero in $R_{2}$ and thus gains zero virtual surplus as well. On the other side, every quantile $q \leq q_{m}\left(R_{1}\right)$ has identical virtual value by construction. We claim that the allocation for each of these quantiles weakly decreases. To see this, note that the allocation of bidding any bid $b \geq v_{m}\left(R_{1}\right)=v_{m}\left(R_{2}\right)$ is the same for both revenue curves $R_{1}$ and $R_{2}$, and the expected payment increases by a constant when the revenue curve $R_{1}$ is replace by $R_{2}$. Thus the agent's preference among all bids $b \geq v_{m}\left(R_{1}\right)$ is the same in both revenue curves $R_{1}$ and $R_{2}$. However, the utility of bidding $b \geq v_{m}\left(R_{2}\right)$ is lower when the revenue curve is $R_{2}$, which implies that there may exist value $v$ such that the agent may prefer bidding 0 to bidding above the monopoly reserve in $R_{2}$, while strictly prefer bidding above the monopoly reserve in $R_{1}$. By Lemma 5.6, the optimal bid for

\footnotetext{
${ }^{11}$ Note that $q_{m} \geq 0.62$ implies that $\hat{q} \geq 0.62$.

${ }^{12}$ By first order condition, for revenue curve $R_{2}$, if $\left(\frac{v}{0.7}-\frac{1}{1-\hat{q}}\right)>1 / \hat{q}$, then bidding $b^{\ddagger}$ already achieves higher utility for the agent compared to bidding below $b^{\ddagger}$. Thus it is sufficient to compare $b^{\ddagger}$ with $\left(\frac{v}{0.7}-\frac{1}{1-\hat{q}}\right)$ in the case that the latter is in $[0,1 / \hat{q}]$.
} 


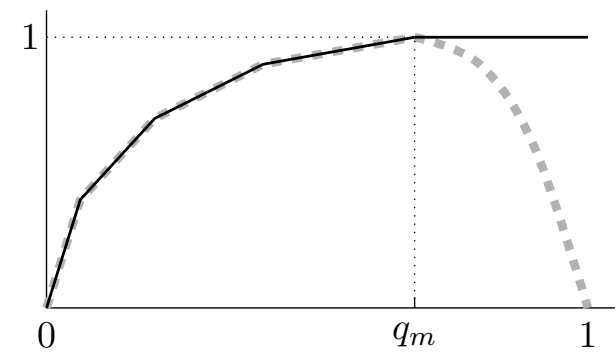

(a) The gray dashed (resp. black solid) curve is the revenue curve $R_{1}$ (resp. $R_{2}$ ). By construction, $q_{m}\left(R_{1}\right)=q_{m}\left(R_{2}\right)$

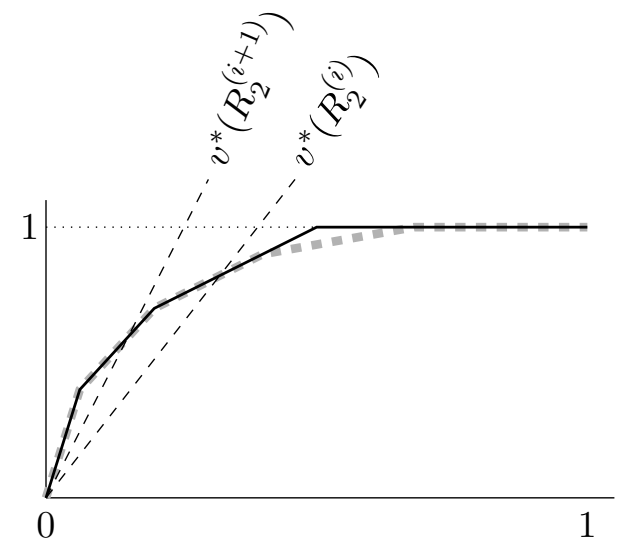

(b) The gray dashed (resp. black solid) curve is revenue curve $R_{2}^{(i)}$ (resp. $R_{2}^{(i+1)}$ ). The slopes of two dotted lines from $(0,0)$ are $v^{*}\left(R_{2}^{(i+1)}\right)$ and $v^{*}\left(R_{2}^{(i)}\right)$ respectively. By construction, $v^{*}\left(R_{2}^{(i+1)}\right) \geq$ $v^{*}\left(R_{2}^{(i)}\right)$.

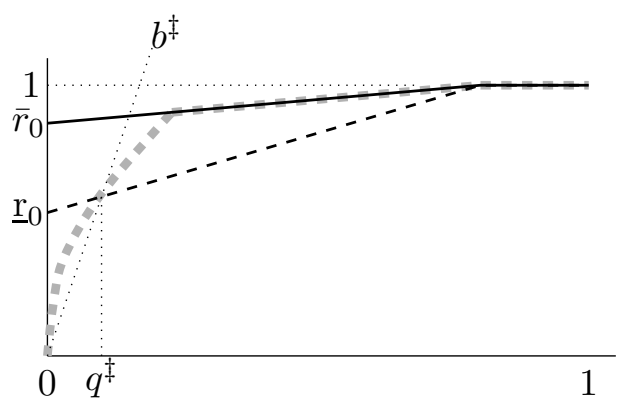

(d) The gray dashed (resp. black solid, black dashed) curve is revenue curve $R_{3}$ (resp. $\left.R_{4}^{\left(\bar{r}_{0}\right)}, R_{4}^{\left(\underline{\mathrm{r}}_{0}\right)}\right)$. The slope of the dotted line from $(0,0)$ is $b^{\ddagger}$, i.e., the optimal bid for an agent with value $v^{*}\left(R_{3}\right)$ and revenue curve $R_{3}$. By construction, $\left.v^{*}\left(R_{4}^{\left(\underline{\mathrm{r}}_{0}\right)}\right) \leq v^{*}\left(R_{3}\right)\right) \leq$ $v^{*}\left(R_{4}^{\left(\bar{r}_{0}\right)}\right)$.

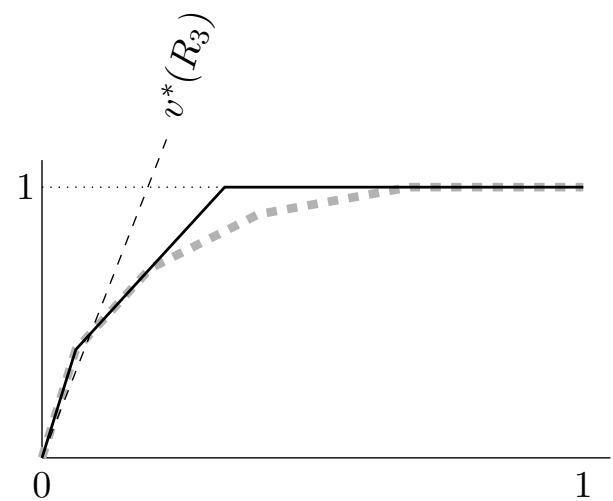

(c) The gray dashed (resp. black solid) curve is revenue curve $R_{2}$ (resp. $R_{3}$ ). The slope of the dotted line from $(0,0)$ is $v^{*}\left(R_{3}\right)$.

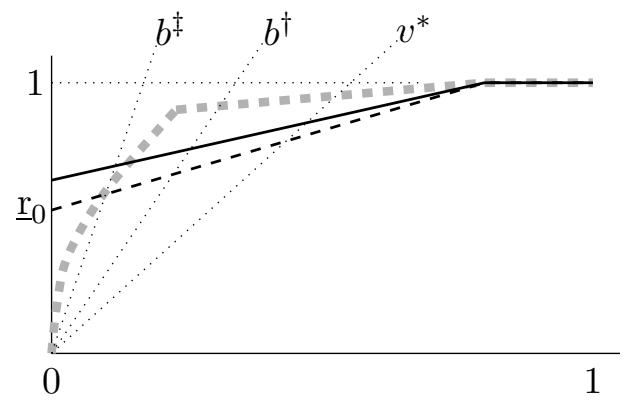

(e) The gray dashed (resp. black solid, black dashed) curve is revenue curve $R_{3}$ (resp. $R_{4}, R_{4}^{\left(\underline{\mathrm{r}}_{0}\right)}$ ). By construction, $v^{*}\left(R_{3}\right)=v^{*}\left(R_{4}\right)\left(\triangleq v^{*}\right)$. The slope of three dotted lines from $(0,0)$ are $b^{\ddagger}, b^{\dagger}$ and $v^{*}$, where $b^{\ddagger}$ (resp. $b^{\dagger}$ ) is the optimal bid for an agent with value $v^{*}$ and revenue curve $R_{3}$ (resp. $R_{4}$ ). By Lemma $3.2 b^{\dagger} \leq b^{\ddagger}$.

Figure 3: Graphical illustration for Lemma 5.7 
any value $v$ is not in $\left(0, v_{m}\left(R_{2}\right)\right)$. Thus, we conclude that $q^{*}\left(R_{2}\right) \leq q^{*}\left(R_{1}\right)$ and (1) the optimal bid (as well as the allocation) for every quantile $q \leq q^{*}\left(R_{2}\right)$ in both $R_{1}$ and $R_{2}$ remains the same; and (2) for every quantile $q \in\left[q^{*}\left(R_{2}\right), q^{*}\left(R_{1}\right)\right)$, the optimal bid quantile $q$ is 0 when the revenue curve is $R_{2}$. This guarantees that the virtual surplus for every quantile $q \leq q_{m}\left(R_{1}\right)$ weakly decreases since the virtual value is non-negative while the allocation decreases. Note that in sample-bid mechanism, the payment for lowest type is always 0, i.e., $p(0)=0$. By Theorem 2.2, the expected revenue (a.k.a. virtual surplus) for $R_{2}$ is at most the expected revenue (a.k.a. virtual surplus) for $R_{1}$.

Step 2- flattening the revenue curve for all quantiles $q \geq q^{*}$. In this step, we start with revenue curve $R_{2}$ constructed in step 1 , and consider a sequence of revenue curves $R_{2}^{(0)}, R_{2}^{(1)}, \ldots$ where $R_{2}^{(0)} \triangleq R_{2}$ and $R_{2}^{(i+1)}$ is recursively defined on $R_{2}^{(i)}$ as follows,

$$
R_{2}^{(i+1)}(q) \triangleq \begin{cases}R_{2}^{(i)}(q) & q \in\left[0, q^{*}\left(R_{2}^{(i)}\right)\right] \\ R_{2}^{(i) \prime}\left(q^{*}\left(R_{2}^{(i)}\right)\right) \cdot\left(q-q^{*}\left(R_{2}^{(i)}\right)\right)+R_{2}^{(i)}\left(q^{*}\left(R_{2}^{(i)}\right)\right) & q \in\left[q^{*}\left(R_{2}^{(i)}\right), \frac{1-R_{2}^{(i)}\left(q^{*}\left(R_{2}^{(i)}\right)\right)}{R_{2}^{(i) \prime}\left(q^{*}\left(R_{2}^{(i)}\right)\right)}+q^{*}\left(R_{2}^{(i)}\right)\right] \\ 1 & q \in\left[\frac{1-R_{2}^{(i)}\left(q^{*}\left(R_{2}^{(i)}\right)\right)}{R_{2}^{(i) \prime}\left(q^{*}\left(R_{2}^{(i)}\right)\right)}+q^{*}\left(R_{2}^{(i)}\right), 1\right]\end{cases}
$$

where $R_{2}^{(i) \prime}\left(q^{*}\left(R_{2}^{(i)}\right)\right)$ is the right-hand derivative of $R_{2}^{(i)}(q)$ at $q=q^{*}\left(R_{2}^{(i)}\right)$. See Figure 3b for a graphical illustration. Invoking Lemma 5.3 and Lemma 5.6, with the same argument for values with positive virtual values in step 1 , we can conclude that $q^{*}\left(R_{2}^{(i)}\right)$ and the expected revenue for $R_{2}^{(i)}$ in the sample-bid mechanism is weakly decreasing in $i$.

Note that by construction, the sequence of revenue curves $R_{2}^{(0)}, R_{2}^{(1)}, \ldots$ converges to a revenue curve $R_{3}$ whose expected revenue in the sample-bid mechanism is at most the revenue for $R_{2}$, and satisfying the following characterization,

$$
R_{3}(q) \triangleq \begin{cases}R_{2}(q) & q \in\left[0, q^{*}\left(R_{3}\right)\right], \\ R_{2}^{\prime}\left(q^{*}\left(R_{3}\right)\right) \cdot\left(q-q^{*}\left(R_{3}\right)\right)+R_{2}\left(q^{*}\left(R_{3}\right)\right) & q \in\left[q^{*}\left(R_{3}\right), \frac{1-R_{2}\left(q^{*}\left(R_{3}\right)\right)}{R_{2}^{\prime}\left(q^{*}\left(R_{3}\right)\right)}+q^{*}\left(R_{3}\right)\right], \\ 1 & q \in\left[\frac{1-R_{2}\left(q^{*}\left(R_{3}\right)\right)}{R_{2}^{\prime}\left(q^{*}\left(R_{3}\right)\right)}+q^{*}\left(R_{3}\right), 1\right] .\end{cases}
$$

See Figure $3 \mathrm{c}$ for a graphical illustration.

Step 3- flattening the revenue curve for all quantile $q \leq q_{m}\left(R_{3}\right)$. For any revenue curve $R$, let $p\left(v^{*}(R), R\right)$ be the expected payment in the sample-bid mechanism of an agent with value $v^{*}(R)$ and revenue curve $R$. Due to Lemma 3.4 and Lemma 3.5, $p\left(v^{*}(R), R\right) \cdot q^{*}(R)$ is a valid lower bound of the expected revenue in the sample-bid mechanism for an agent with revenue curve $R$. In this step, instead of analyzing the expected revenue, we argue that we can convert any revenue curve $R_{3}$ (constructed in step 2) into another revenue curve $R_{4}$, such that (i) $v^{*}\left(R_{4}\right)=v^{*}\left(R_{3}\right)\left(\triangleq v^{*}\right)$; (ii) $q^{*}\left(R_{4}\right) \leq q^{*}\left(R_{3}\right)$; and (iii) $p\left(v^{*}, R_{4}\right) \leq p\left(v^{*}, R_{3}\right)$. Finally, by showing that $p\left(v^{*}\left(R_{4}\right), R_{4}\right) \cdot q^{*}\left(R_{4}\right) \geq$ 0.545 , we finish the proof of the lemma.

Given the revenue curve $R_{3}$ constructed in step 2, for any $r_{0} \in[0,1]$, we define a revenue curve 
$R_{4}^{\left(r_{0}\right)}$ as follows,

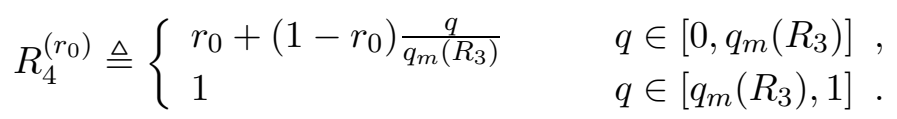

See the black curves in Figure $3 \mathrm{~d}]$ as an example. We claim that there exists $r_{0}^{*} \in[0,1]$ s.t. $R_{4}^{(r 0)}\left(\triangleq R_{4}\right.$ ) satisfies properties (i) (ii) (iii) mentioned above. To see this, consider the argument as follows.

By construction, for all every value $v$, every bid $b$, the utility $u\left(v, b, R_{4}^{\left(r_{0}\right)}\right)$ is decreasing continuously in $r_{0}$. Thus, $v^{*}\left(R_{4}^{\left(r_{0}\right)}\right)$ is decreasing continuously in $r_{0}$. Let $b^{\ddagger}$ be the optimal bid of an agent with value $v^{*}\left(R_{3}\right)$ and revenue curve $R_{3}$. Denote $q\left(b^{\ddagger}, R_{3}\right)$ by $q^{\ddagger}$. Consider revenue curve $R_{4}^{\left(\underline{\mathrm{r}}_{0}\right)}$ where $\underline{\mathrm{r}}_{0} \triangleq 1-\frac{q_{m}\left(R_{3}\right)}{q_{m}\left(R_{3}\right)-q^{\ddagger}}\left(1-R_{3}\left(q^{\ddagger}\right)\right)$. By construction, $R_{4}^{\left(\underline{\mathrm{r}}_{0}\right)}(q) \geq R_{3}(q)$ for all $q \leq q^{\ddagger}$, and $R_{4}^{\left(\underline{\mathrm{r}}_{0}\right)}(q) \leq R_{3}(q)$ for all $q \geq q^{\ddagger}$. See Figure $3 \mathrm{~d}$ for a graphical illustration. Note that by construction,

$$
\begin{aligned}
u\left(v^{*}\left(R_{3}\right), b^{\ddagger}, R_{4}^{\left(\mathrm{r}_{0}\right)}\right) & =v^{*}\left(R_{3}\right) \cdot\left(1-q^{\ddagger}\right)-\alpha b^{\ddagger} \cdot q^{\ddagger}-\alpha \int_{q^{\ddagger}}^{1} \frac{R_{4}^{\left(\underline{\mathrm{r}}_{0}\right)}(q)}{q} d q \\
& \geq v^{*}\left(R_{3}\right) \cdot\left(1-q^{\ddagger}\right)-\alpha b^{\ddagger} \cdot q^{\ddagger}-\alpha \int_{q^{\ddagger}}^{1} \frac{R_{3}(q)}{q} d q=u\left(v^{*}\left(R_{3}\right), b^{\ddagger}, R_{3}\right)=0
\end{aligned}
$$

Thus, $v^{*}\left(R_{4}^{\left(\underline{\mathrm{r}}_{0}\right)}\right) \leq v^{*}\left(R_{3}\right)$. Next, consider revenue curve $R_{4}^{\left(\bar{r}_{0}\right)}$ where $\bar{r}_{0} \triangleq 1-\frac{q_{m}\left(R_{3}\right)}{q_{m}\left(R_{3}\right)-q^{*}\left(R_{3}\right)}(1-$ $R_{3}\left(q^{*}\left(R_{3}\right)\right)$. By construction, $R_{4}^{\left(\bar{r}_{0}\right)}(q) \geq R_{3}(q)$ for all $q \in[0,1]$. See Figure $3 \mathrm{~d}$ for a graphical illustration. Thus, $v^{*}\left(R_{4}^{\left(\bar{r}_{0}\right)}\right) \geq v^{*}\left(R_{3}\right)$ with the similar argument for $R_{4}^{\left(\underline{\mathrm{r}}_{0}\right)}$ Therefore, we know that there exists $r_{0}^{*} \in\left[\underline{\mathrm{r}}_{0}, \bar{r}_{0}\right]$ such that $v^{*}\left(R_{4}^{\left(r_{0}^{*}\right)}\right)=v^{*}\left(R_{3}\right)$. We denote $R_{4}^{\left(r_{0}^{*}\right)}$ by $R_{4}$ and show that $R_{4}$ satisfies properties (ii) $q^{*}\left(R_{4}\right) \leq q^{*}\left(R_{3}\right)$ and (iii) $p\left(v^{*}, R_{4}\right) \leq p\left(v^{*}, R_{3}\right)$ with the argument below.

Lemma 5.3 implies that $b^{\ddagger}>v^{*}\left(R_{3}\right)$. Combining with the fact that $r_{0}^{*} \geq \underline{\mathrm{r}}_{0}$, we know that property (ii) $q^{*}\left(R_{4}\right) \leq q^{*}\left(R_{3}\right)$ is satisfied. See Figure $3 \mathrm{e}$ for a graphical illustration.

Combining the first order condition in Lemma 3.2 and construction of $R_{4}$, it is guaranteed that the optimal bid $b^{\dagger}$ of value $v^{*}$ for revenue curve $R_{4}$ is at most $b^{\ddagger}$. Furthermore, $q\left(b^{\dagger}, R_{4}\right) \geq q\left(b^{\ddagger}, R_{4}\right) \geq$ $q\left(b^{\ddagger}, R_{3}\right)=q^{\ddagger}$ by construction. By the definition, the optimal utility of value $v^{*}(R)$ for any revenue curve $R$ is zero. Thus, $p\left(v^{*}, R_{3}\right)=v^{*} \cdot\left(1-q^{\ddagger}\right) \geq v^{*} \cdot\left(1-q\left(b^{\dagger}, R_{4}\right)\right)=p\left(v^{*}, R_{4}\right)$.

Step 4- lower-bounding the expected revenue on $R_{4}$. So far, we have shown that for an arbitrary revenue curve satisfying the assumptions in lemma statement, its expected revenue in the sample-bid mechanism is lower-bounded by $p\left(v^{*}\left(R_{4}\right), R_{4}\right) \cdot q^{*}\left(R_{4}\right)$ for $R_{4}$ pinned down by some $\left(r_{0}, q_{m}\right)$ as follows,

$$
R_{4} \triangleq \begin{cases}r_{0}+\left(1-r_{0}\right) \frac{q}{q_{m}} & q \in\left[0, q_{m}\right] \\ 1 & q \in\left[q_{m}, 1\right] .\end{cases}
$$

By numerically verifying $p\left(v^{*}\left(R_{4}\right), R_{4}\right) \cdot q^{*}\left(R_{4}\right) \geq 0.545$ for all $\left(r_{0}, q_{m}\right) \in[0,1]^{2}$, we finish the proof. The details of this numerical evaluation is elaborated on in Appendix A.

\subsection{Regular Distributions with Monopoly Quantile $q_{m} \leq 0.62$}

In this subsection, we analyze the prior-independent approximation ratio of the sample-bid mechanism over the class of regular distributions with monopoly quantile $q_{m} \leq 0.62$. 


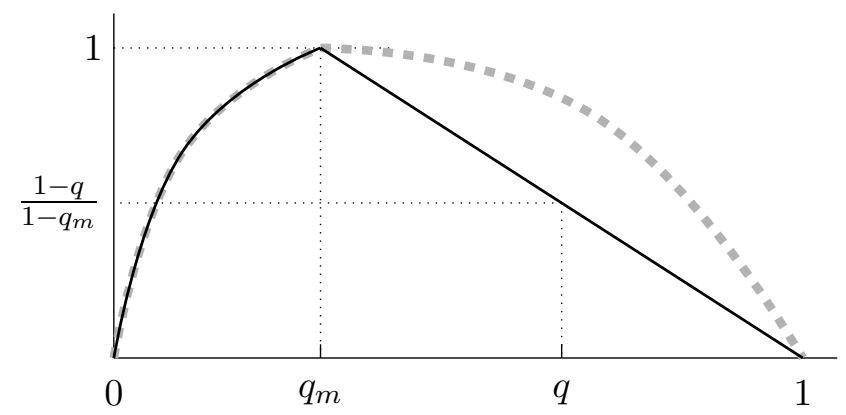

Figure 4: Graphical illustration for Lemma 5.9. The gray dashed (resp. black solid) curve is revenue curve $R$ (resp. lower bound of $R$ ).

Lemma 5.8. For the sample-bid mechanism with $\alpha=0.7$, the prior-independent approximation ratio over the class of regular distributions with monopoly quantile $q_{m} \leq 0.62$ is at most 1.835 .

Fix an arbitrary revenue curve $R$, let

$$
v^{*}(R) \triangleq \inf \left\{v: b(v, R) \geq v_{m}(R)\right\}
$$

be the smallest value whose optimal bid $b(v, R)$ for revenue curve $R$ is at least $v_{m}(R)$. Since Lemma 3.5 guarantees that $b(v, R)$ is weakly non-decreasing in $v, v^{*}(R)$ is well-defined, $b(v, R) \geq$ $v_{m}(R)$ for all $v \geq v^{*}(R)$. Furthermore, by Lemma 5.3, we know that $b(v, R) \geq v_{m}(R) / 0.7$ for all $v \geq$ $\max \left\{v^{*}(R), v_{m}(R)\right\}$. Denote $q\left(v^{*}(R), R\right)$ by $q^{*}(R)$. By Lemma 3.4 and Lemma 3.5, the expected revenue $\boldsymbol{R e v}_{R}(\mathrm{SB})$ of the sample-bid mechanism for revenue curve $R$ can be lower-bounded as follows,

$$
\begin{aligned}
\operatorname{Rev}_{R}(\mathrm{SB})= & \int_{0}^{1} p(v(q, R), R) d q \\
= & \int_{0}^{\min \left\{q^{*}(R), q_{m}(R)\right\}} p(v(q, R), R) d q \\
& +\int_{\min \left\{q^{*}(R), q_{m}(R)\right\}}^{q^{*}(R)} p(v(q, R), R) d q \\
& +\int_{q^{*}(R)}^{1} p(v(q, R), R) d q \\
\geq \tilde{p}\left(v_{m}(R) / 0.7, R\right) \cdot \min \left\{q^{*}(R), q_{m}(R)\right\} & +\tilde{p}\left(v_{m}(R), R\right) \cdot \max \left\{0, q^{*}(R)-q_{m}(R)\right\} \\
& +\int_{q^{*}(R)}^{1} p(v(q, R), R) d q .
\end{aligned}
$$

Denote $q\left(v_{m}(R) / 0.7, R\right)$ by $q^{\ddagger}(R)$, and $\int_{q^{\ddagger}(R)}^{q_{m}(R)} \frac{R(q)}{q} d q$ by $w(R)$. In Lemma [5.9, we lower-bound the expected payment $\tilde{p}\left(v_{m}(R) / 0.7, R\right)$ and $\tilde{p}\left(v_{m}(R), R\right)$ as the function of $q_{m}(R), q^{\ddagger}(R), w(R)$ and $v^{*}(R)$. In Lemma 5.10, we lower-bound $q^{*}(R)$ as the function of $q_{m}(R), q^{\ddagger}(R)$ and $v^{*}(R)$. In Lemma 5.11, we upper-bound of $v^{*}(R)$ as the function of $q_{m}(R), q^{\ddagger}(R)$ and $w(R)$. In Lemma 5.12, we lowerbound $p(v(q, R), R)$ as a function of $q_{m}(R)$ for all quantile $q \in\left[q_{m}(R), 1\right]$. Putting all pieces together, we show Lemma 5.8 by providing a lower bound of expected revenue in the sample-bid mechanism as a function of $q_{m}(R), q^{\ddagger}(R)$ and $w(R)$, and numerically evaluating its value for all possible parameters. The details of the numerical evaluations in this section are similar to those of Lemma 5.7, which are elaborated on in Appendix A,

Lemma 5.9. For the sample-bid mechanism with $\alpha=0.7$, given any concave revenue curve $R$, the 
expected payment $\tilde{p}(b, R)$ of bidding $b \in\left[0, v_{m}(R)\right]$ is at least

$$
\tilde{p}(b, R) \geq \frac{0.7 \log \left(b \cdot\left(1-q_{m}(R)\right)+1\right)}{1-q_{m}(R)} ;
$$

and the expected payment $\tilde{p}\left(v_{m}(R) / 0.7, R\right)$ of bidding $v_{m}(R) / 0.7$ is at least

$$
\tilde{p}\left(v_{m}(R) / 0.7, R\right) \geq\left(\frac{q^{\ddagger}(R)}{q_{m}(R)}+0.7 w(R)-\frac{\left.0.7 \log \left(q_{m}(R)\right)\right)}{1-q_{m}(R)}\right) .
$$

Proof. By definition, for any $b \in\left[0, v_{m}(R)\right]$,

$$
\begin{aligned}
\tilde{p}(b, R) & =0.7 b \cdot q(b, R)+0.7 \int_{q(b, R)}^{1} \frac{R(q)}{q} d q \\
& \geq 0.7 b \cdot q(b, R)+0.7 \int_{q(b, R)}^{1} \frac{\frac{1-q}{1-q_{m}(R)}}{q} d q \\
& =0.7 b \cdot q(b, R)-0.7 \frac{1-q(b, R)}{1-q_{m}(R)}-\frac{0.7 \log (q(b, R))}{1-q_{m}(R)} \\
& \geq \frac{0.7 \log \left(b \cdot\left(1-q_{m}(R)\right)+1\right)}{1-q_{m}(R)}
\end{aligned}
$$

where the first inequality uses the fact that $R(q) \geq \frac{1-q}{1-q_{m}(R)}$ for all $q \geq q_{m}(R)$ from the regularity of $R$, and the second inequality use the fact that $b \cdot q(b, R) \geq \frac{1-q(b, R)}{1-q_{m}(R)}$, and $q(b, R) \leq\left(b \cdot\left(1-q_{m}(R)\right)+\right.$ $1)^{-1}$ from the regularity of $R$. See Figure 4 for a graphical illustration.

Similarly,

$$
\begin{aligned}
\tilde{p}\left(v_{m}(R) / 0.7, R\right) & =0.7 \frac{v_{m}(R)}{0.7} q^{\ddagger}(R)+0.7 \int_{q^{\ddagger}(R)}^{1} \frac{R(q)}{q} d q \\
& =\frac{q^{\ddagger}(R)}{q_{m}(R)}+0.7 w(R)+0.7 \int_{q_{m}(R)}^{1} \frac{R(q)}{q} d q \\
& \geq \frac{q^{\ddagger}(R)}{q_{m}(R)}+0.7 w(R)-\frac{0.7 \log \left(q_{m}(R)\right)}{1-q_{m}(R)} .
\end{aligned}
$$

Lemma 5.10. For any concave revenue curve $R$, the quantile $q(v, R)$ for value $v \leq v_{m}(R)$ is at least

$$
q(v, R) \geq \frac{1}{1+v \cdot\left(1-q_{m}(R)\right)}
$$

and the quantile $q(v, R)$ for value $v \in\left[v_{m}(R), v_{m}(R) / 0.7\right]$ is at least

$$
q(v, R) \geq \frac{2 q_{m}(R)-q^{\ddagger}(R) \cdot(1+1 / 0.7)}{1+v \cdot\left(1-q_{m}(R)\right)} .
$$

Proof. Given any concave revenue curve $R_{1}$, consider another revenue curve $R_{2}$ defined as follows,

$$
R_{2}(q) \triangleq \begin{cases}R_{1}(q) & q \in\left[0, q^{\ddagger}\left(R_{1}\right)\right], \\ R_{1}\left(q^{\ddagger}\left(R_{1}\right)\right)+\frac{q-q^{\ddagger}\left(R_{1}\right)}{q_{m}\left(R_{1}\right)-q^{\ddagger}\left(R_{1}\right)}\left(1-R_{1}\left(q^{\ddagger}\left(R_{1}\right)\right)\right) & q \in\left[q^{\ddagger}\left(R_{1}\right), q_{m}\left(R_{1}\right)\right], \\ \frac{1-q}{1-q_{m}\left(R_{1}\right)} & q \in\left[q_{m}\left(R_{1}\right), 1\right] .\end{cases}
$$




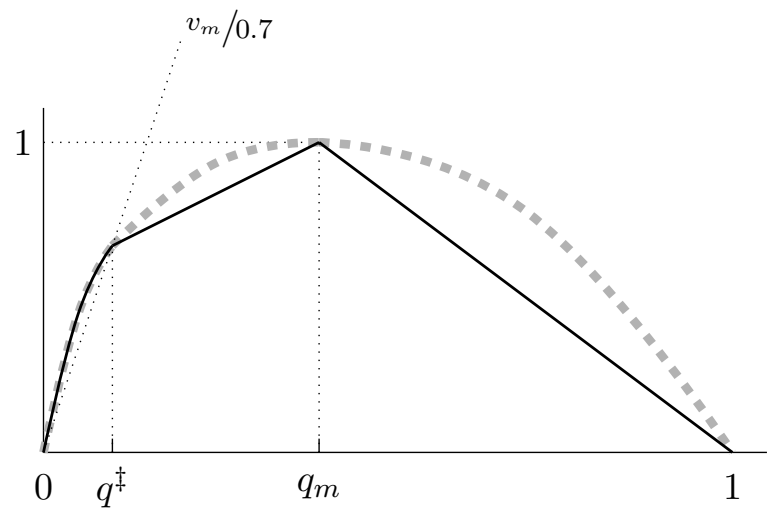

Figure 5: Graphical illustration for Lemma 5.10, The gray dashed (resp. black solid) curve is revenue curve $R_{1}$ (resp. $R_{2}$ ). The slope of the dotted line from $(0,0)$ is $v_{m}\left(R_{1}\right) / 0.7$.

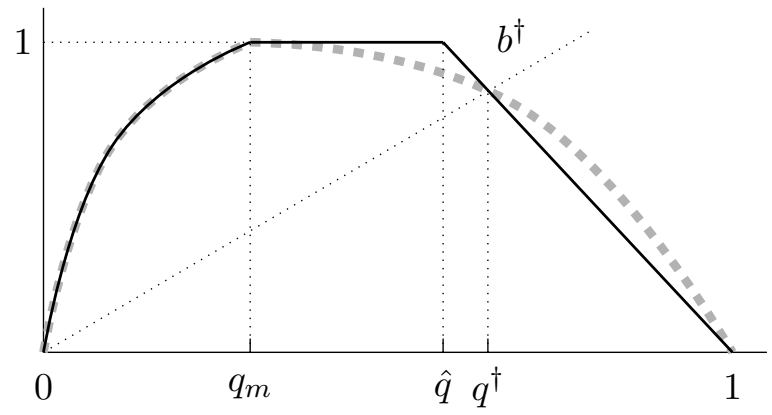

Figure 6: Graphical illustration for Lemma 5.11, The gray dashed (resp. black solid) curve is revenue curve $R_{1}$ (resp. $R_{2}$ ). The slope of the dotted line from $(0,0)$ is $b^{\dagger}$.

Since $R_{1}$ is regular, we have $R_{2}(q) \leq R_{1}(q)$ for all $q \in[0,1]$ by construction. See Figure 5 for graphical illustration. Thus, for any value $v \leq v_{m} R_{1}$, we have

$$
q\left(v, R_{1}\right) \geq q\left(v, R_{2}\right)=\frac{1}{1+v \cdot\left(1-q_{m}\left(R_{1}\right)\right)} .
$$

Moreover, for any value $v \in\left[v_{m}\left(R_{1}\right), v_{m}\left(R_{1}\right) / 0.7\right]$, we have

$$
q\left(v, R_{1}\right) \geq q\left(v, R_{2}\right)=\frac{2 q_{m}\left(R_{1}\right)-q_{1}\left(R_{1}\right) \cdot(1+1 / 0.7)}{1+v \cdot\left(1-q_{m}\left(R_{1}\right)\right)} .
$$

Lemma 5.11. In the sample-bid mechanism with parameter $\alpha=0.7$, given any value $v$ and any concave revenue curve $R$, the optimal bid $b(v, R)$ for an agent with value $v$ and revenue curve $R$ is at least $v_{m}(R)$ if for all $\hat{q} \in\left[q_{m}(R), 1\right]$,

$$
\begin{aligned}
v \cdot\left(1-q^{\ddagger}(R)\right)-v_{m}(R) \cdot q^{\ddagger}(R)-0.7\left(w(R)+\log \left(\frac{\hat{q}}{q_{m}(R)}\right)\right. & \left.-\frac{\ln (\hat{q})}{1-\hat{q}}\right) \\
& \geq v(1-\tilde{q})+\frac{0.7 \log (\tilde{q})}{1-\hat{q}}
\end{aligned}
$$

where $\tilde{q} \triangleq\left(1+\min \left\{1 / \hat{q}, \max \left\{0, \frac{v}{0.7}-\frac{1}{1-\hat{q}}\right\}\right\} \cdot(1-\hat{q})\right)^{-1}$. 
Proof. Fix an arbitrary concave revenue curve $R$. We show that inequality (2) in the lemma statement is a sufficient condition that bidding $v_{m}(R) / 0.7$ is weakly preferred than bidding any bids in $\left[0, v_{m}(R)\right]$. The argument is similar to Lemma 5.6.

We prove by contradiction, suppose there exists an revenue curve $R_{1}$, and value $v$ such that inequality (2) in the lemma statement is satisfied but the optimal bid of an agent with value $v$ and revenue curve $R_{1}$ is $b^{\dagger} \in\left[0, v_{m}\left(R_{1}\right)\right)$. Denote $q\left(b^{\dagger}, R_{1}\right)$ by $q^{\dagger}$. Let $\hat{q} \triangleq 1-\frac{1-q^{\dagger}}{R_{1}\left(q^{\dagger}\right)}$. By construction, $\hat{q} \geq q_{m}\left(R_{1}\right)$. Now consider another revenue curve $R_{2}$ defined as follows,

$$
R_{2}(q) \triangleq \begin{cases}R_{1}(q) & q \in\left[0, q_{m}\left(R_{1}\right)\right] \\ 1 & q \in\left[q_{m}\left(R_{1}\right), \hat{q}\right] \\ \frac{1-q}{1-\hat{q}} & q \in[\hat{q}, 1] .\end{cases}
$$

By construction, $R_{2}$ is a concave revenue curve s.t. (i) $R_{1}(q)=R_{2}(q)$ for all $q \in\left[0, q_{m}\left(R_{1}\right)\right]$; (ii) $R_{1}(q) \leq R_{2}(q)$ for all $q \in\left[q_{m}\left(R_{1}\right), q^{\dagger}\right]$; and (iii) $R_{1}(q) \geq R_{2}(q)$ for all $q \in\left[q^{\dagger}, 1\right]$; See Figure 6 for a graphical illustration.

Applying Lemma 5.2 on $R_{1}, R_{2}, q^{\dagger}, v$ and all $b^{\ddagger} \geq b^{\dagger}$, we conclude that the optimal bid for an agent with value $v$ and revenue curve $R_{2}$ is in $\left[0, b^{\dagger}\right]$.

Note that for any bid $b \in[0,1 / \hat{q}]$, the utility $u\left(v, b, R_{2}\right)$ has a closed-form expression as follows,

$$
u\left(v, b, R_{2}\right)=v \cdot \frac{b(1-\hat{q})}{b(1-\hat{q})+1}+0.7 \log \left(\frac{1}{b(1-\hat{q})+1}\right) .
$$

Considering the first order condition of $u\left(v, b, R_{2}\right)$ with respect to bid $b$, after basic simplification, we have

$$
b=\frac{v}{0.7}-\frac{1}{1-\hat{q}}
$$

Thus, the optimal bid in $[0,1 / \hat{q}]$ for revenue curve $R_{2}$ is $\tilde{b} \triangleq \min \left\{1 / \hat{q}, \max \left\{0, \frac{v}{0.7}-\frac{1}{1-\hat{q}}\right\}\right\}$. Plugging $u\left(v, b, R_{2}\right)$ with $b=\tilde{b}$, we get

$$
v(1-\tilde{q})+\frac{0.7 \log (\tilde{q})}{1-\hat{q}}
$$

i.e., the right hand side of inequality (2).

Moreover, note that the utility $u\left(v, v_{m}\left(R_{1}\right) / 0.7, R_{2}\right)$ has a closed-form expression as follows,

$$
v \cdot\left(1-q^{\ddagger}(R)\right)-v_{m}(R) \cdot q^{\ddagger}(R)-0.7\left(w(R)+\log \left(\frac{\hat{q}}{q_{m}(R)}\right)-\frac{\ln (\hat{q})}{1-\hat{q}}\right)
$$

i.e., the left hand side of inequality (2). This leads to a contradiction, which finishes the proof.

Definition 5.2. A pentagon revenue curve $R$ parameterized by the quantile $q_{k} \in\left[q_{m}(R), 1\right]$ of kink and the revenue $r_{k} \in\left[\frac{1-q_{k}}{1-q_{m}(R)}, 1\right]$ on this kink is defined as follows

$$
R(q) \triangleq \begin{cases}1 & q \in\left[0, q_{m}(R)\right], \\ r_{k}+\frac{q-q_{m}(R)}{q_{k}-q_{m}(R)}\left(1-r_{k}\right) & q \in\left[q_{m}(R), q_{k}\right], \\ \frac{1-q}{1-q_{k}} \cdot r_{k} & q \in\left[q_{k}, 1\right] .\end{cases}
$$




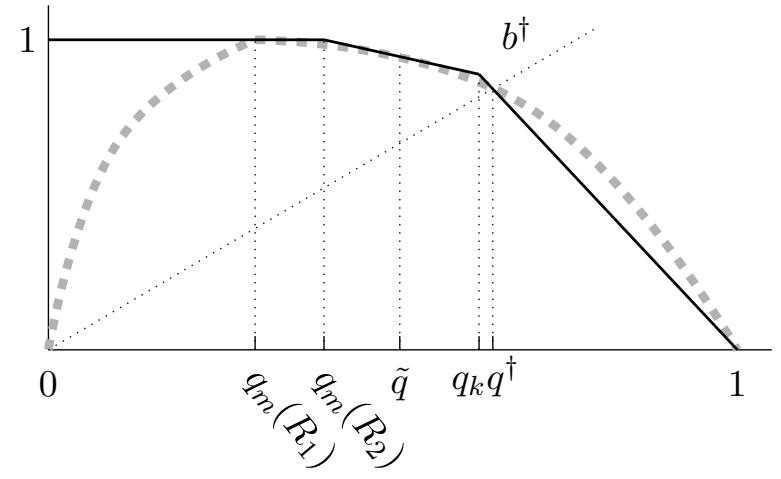

(a) Case (i) $b^{\dagger} \leq \tilde{v}$.

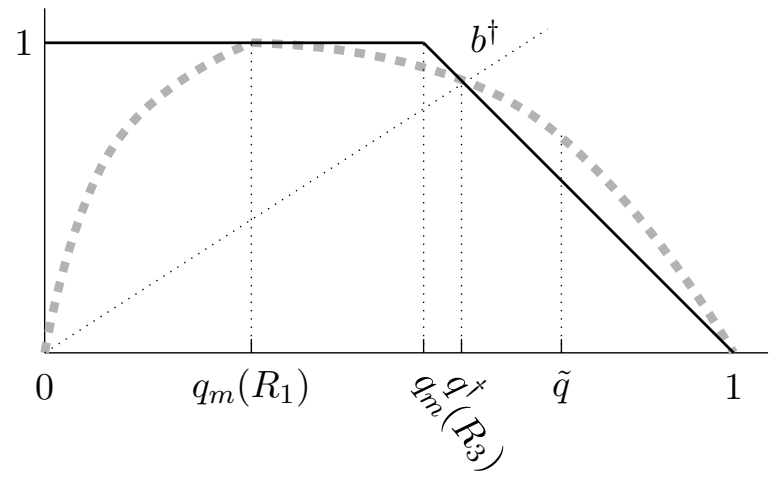

(b) Case (ii) $b^{\dagger} \geq \tilde{v}$.

Figure 7: Graphical illustration for Lemma 5.12. The gray dashed (resp. black solid) curve is revenue curve $R_{1}$ (resp. $R_{2}$ in (a) and $R_{3}$ in (b)). The slope of the dotted line from $(0,0)$ is $b^{\dagger}$.

An example of a pentagon revenue curve is illustrated as the solid curve in Figure 7a as the solid line.

Lemma 5.12. In the sample-bid mechanism, given any quantile $\hat{q} \in[0,1]$, quantile $\tilde{q} \in[\hat{q}, 1]$, and bid $b \in[0,1 / \hat{q}]$, if for all pentagon revenue curves $R_{P}$ with $q_{m}\left(R_{P}\right) \geq \hat{q}$, the optimal bid of an agent with value $v\left(\tilde{q}, R_{P}\right)$ and revenue curve $R_{P}$ is at least $b$; then for all concave revenue curves $R$ with $q_{m}(R)=\hat{q}$, the optimal bid of an agent with value $v(\tilde{q}, R)$ and revenue curve $R$ is at least $b$ as well.

Proof. Fix arbitrary $\hat{q} \in[0,1], \tilde{q} \in[\hat{q}, 1]$, and concave revenue curve $R_{1}$ with $q_{m}\left(R_{1}\right)=\hat{q}$. Let $b^{\dagger}$ be the optimal bid for an agent with value $v\left(\tilde{q}, R_{1}\right)(\triangleq \tilde{v})$ and revenue curve $R_{1}$. To show this lemma, it is sufficient to assume $b^{\dagger} \leq 1 / q_{m}\left(R_{1}\right)$. Now we consider two cases, i.e., $b^{\dagger} \leq \tilde{v}$ and $b^{\dagger} \geq \tilde{v}$ separately.

Case (i) $b^{\dagger} \leq \tilde{v}$ : Consider the pentagon revenue curve $R_{2}$ with

$$
\begin{gathered}
q_{m}\left(R_{2}\right)=\tilde{q}+\frac{1-R_{1}(\tilde{q})}{R_{1}^{\prime}(\tilde{q})}, \quad q_{k}=\frac{\tilde{q} R_{1}^{\prime}(\tilde{q})-R_{1}(\tilde{q})+\frac{R_{1}\left(q^{\dagger}\right)}{1-q^{\dagger}}}{R_{1}^{\prime}(\tilde{q})+\frac{R_{1}\left(q^{\dagger}\right)}{1-q^{\dagger}}}, \\
r_{k}=\frac{1-q_{k}}{1-q^{\dagger}} R_{1}\left(q^{\dagger}\right) .
\end{gathered}
$$

where $R_{1}^{\prime}(\tilde{q})$ is the right-hand derivative of $R_{1}(q)$ at $q=\tilde{q}$. By construction, we have (i) $R_{2}(\tilde{q})=$ $R_{1}(\tilde{q})$ and thus $v\left(\tilde{q}, R_{2}\right)=v\left(\tilde{q}, R_{1}\right)=\tilde{v}$; (ii) $R_{2}\left(q^{\dagger}\right)=R_{1}\left(q^{\dagger}\right)$; and (iii) $R_{2}(q) \geq R_{1}(q)$ for all $q \in\left[0, q^{\dagger}\right]$. See Figure 7 a for a graphical illustration.

Applying Lemma 5.2 on $R_{1}, R_{2}, q^{\dagger}, \tilde{v}$ and all $b^{\ddagger} \geq b^{\dagger}$, we conclude that the optimal bid for value $\tilde{v}$ is weakly smaller than $b^{\dagger}$. Thus, for any bid $b \in[0,1 / \hat{q}]$, if the optimal bid for value $v\left(\tilde{q}, R_{2}\right)$ in revenue curve $R_{2}$ is at least $b$, then the optimal bid $b^{\dagger}$ for value $v\left(\tilde{q}, R_{1}\right)$ in revenue curve $R_{1}$ is at least $b$ as well. 
Case (ii) $b^{\dagger} \geq \tilde{v}$ : Consider the pentagon revenue curve $R_{3}$ with

$$
q_{m}\left(R_{3}\right)=1-\frac{1-q^{\dagger}}{R_{1}\left(q^{\dagger}\right)}, \quad q_{k}=q_{m}\left(R_{3}\right), \quad r_{k}=1 .
$$

By construction, we have (i) $q\left(\tilde{v}, R_{3}\right) \leq q\left(\tilde{v}, R_{1}\right)$ and thus $v\left(\tilde{q}, R_{3}\right) \leq v\left(\tilde{q}, R_{1}\right)$; (ii) $R_{3}\left(q^{\dagger}\right)=R_{1}\left(q^{\dagger}\right)$; and (iii) $R_{3}(q) \geq R_{1}(q)$ for all $q \in\left[0, q^{\dagger}\right]$. See Figure $7 \mathrm{~b}$ for a graphical illustration.

Applying Lemma 5.2 on $R_{1}, R_{3}, q^{\dagger}, \tilde{v}$ and all $b^{\ddagger} \geq b^{\dagger}$, we conclude that the optimal bid for value $\tilde{v}$ is weakly smaller than $b^{\dagger}$. Thus, for any bid $b \in[0,1 / \hat{q}]$, if the optimal bid for value $v\left(\tilde{q}, R_{3}\right)$ in revenue curve $R_{3}$ is at least $b$, then combining with Lemma 3.5, the optimal bid $b^{\dagger}$ for value $v\left(\tilde{q}, R_{1}\right)$ in revenue curve $R_{1}$ is at least $b$ as well.

Now we are ready to prove Lemma 5.8 .

Proof of Lemma 5.8. Fix an arbitrary concave revenue curve $R$ with $q_{m}(R) \leq 0.62$. We consider $v^{*}(R) \leq v_{m}(R), v_{m}(R) \leq v^{*}(R) \leq v_{m}(R) / 0.7$, and $v^{*}(R) \geq v_{m}(R) / 0.7$ separately.

Case (i) $v^{*}(R) \leq v_{m}(R)$ : By Lemma 3.4 and Lemma 3.5, the expected revenue $\boldsymbol{R e v}_{R}(\mathrm{SB})$ of the sample-bid mechanism for revenue curve $R$ can be lower-bounded as follows,

$$
\begin{aligned}
\operatorname{Rev}_{R}(\mathrm{SB}) & =\int_{0}^{1} p(v(q, R), R) d q \\
& =\int_{0}^{q_{m}(R)} p(v(q, R), R) d q+\int_{q_{m}(R)}^{q^{*}(R)} p(v(q, R), R) d q+\int_{q^{*}(R)}^{1} p(v(q, R), R) d q \\
& \geq \tilde{p}\left(v_{m}(R) / 0.7, R\right) \cdot q_{m}(R)+\tilde{p}\left(v_{m}(R), R\right) \cdot\left(q^{*}(R)-q_{m}(R)\right)+\int_{q^{*}(R)}^{1} p(v(q, R), R) d q .
\end{aligned}
$$

Invoking Lemma 5.9 and Lemma 5.10 , we can rewrite the lower bound of $\operatorname{Rev}[R]$ as

$$
\begin{aligned}
\geq( & \left.\frac{q^{\ddagger}(R)}{q_{m}(R)}+0.7 w(R)-\frac{\left.0.7 \log \left(q_{m}(R)\right)\right)}{1-q_{m}(R)}\right) \cdot q_{m}(R) \\
& \quad-\frac{0.7 \log \left(q_{m}(R)\right)}{1-q_{m}(R)} \cdot\left(\frac{1}{1-v^{*}(R) \cdot\left(1+q_{m}(R)\right)}-q_{m}(R)\right)+\int_{q^{*}(R)}^{1} p(v(q, R), R) d q .
\end{aligned}
$$

Note that this lower bound is weakly decreasing in $v^{*}(R)$ while holding everything else fixed. Let $v^{*}\left(q_{m}(R), q^{\ddagger}(R), w(R)\right)$ be the upper bound of $v^{*}(R)$ as the function of $q_{m}(R), q^{\ddagger}(R), w(R)$ established in Lemma 5.11. From Lemma 5.10, we can lower bound $\left.v^{*}\left(q_{m}(R), q^{\ddagger}(R), w(R)\right)\right)$ by $q^{*}\left(q_{m}(R), q^{\ddagger}(R), w(R)\right) \triangleq\left(1-v^{*}\left(q_{m}(R), q^{\ddagger}(R), w(R)\right) \cdot\left(1+q_{m}(R)\right)\right)^{-1}$. Let $b\left(q, q_{m}(R)\right)$ be the lower bound of the optimal bid for an agent with value $v(q, R)$ and revenue curve $R$ as the function of $q, q_{m}(R)$ established in Lemma 5.12 . Then, we can further rewrite the lower bound of $\operatorname{Rev}[R]$ as

$$
\begin{aligned}
& \geq\left(\frac{q^{\ddagger}(R)}{q_{m}(R)}+0.7 w(R)-\frac{\left.0.7 \log \left(q_{m}(R)\right)\right)}{1-q_{m}(R)}\right) \cdot q_{m}(R) \\
& \quad-\frac{0.7 \log \left(q_{m}(R)\right)}{1-q_{m}(R)} \cdot\left(q^{*}\left(q_{m}(R), q^{\ddagger}(R), w(R)\right)-q_{m}(R)\right) \\
& \quad+\int_{q^{*}\left(q_{m}(R), q^{\ddagger}(R), w(R)\right)}^{1} \frac{0.7 \log \left(b\left(q, q_{m}(R)\right) \cdot\left(1-q_{m}(R)\right)+1\right)}{1-q_{m}(R)} d q .
\end{aligned}
$$


where the bid $b\left(q, q_{m}(R)\right)$ in the last term can be lower-bounded using Lemma 5.9.

Therefore, we lower-bound $\boldsymbol{R e v}_{R}(\mathrm{SB})$ as the function of $q_{m}(R), q^{\ddagger}(R), w(R)$. By numerically enumerating all possible parameters, we conclude that $\boldsymbol{R e v}_{R}(\mathrm{SB}) \geq 0.545$ in this case.

Case (ii) $v_{m}(R) \leq v^{*}(R) \leq v_{m}(R) / 0.7$ : The analysis is similar to case (i). By Lemma 3.4 and Lemma 3.5, the expected revenue $\operatorname{Rev}_{R}(\mathrm{SB})$ of the sample-bid mechanism for revenue curve $R$ can be lower-bounded as follows,

$$
\begin{aligned}
\operatorname{Rev}_{R}(\mathrm{SB}) & =\int_{0}^{1} p(v(q, R), R) d q \\
& \geq \int_{0}^{q^{*}(R)} p(v(q, R), R) d q+\int_{q_{m}(R)}^{1} p(v(q, R), R) d q \\
& \geq \tilde{p}\left(v_{m}(R) / 0.7, R\right) \cdot q^{*}(R)+\int_{q_{m}(R)}^{1} p(v(q, R), R) d q
\end{aligned}
$$

Invoking Lemma 5.9 and Lemma 5.10 , we can rewrite the lower bound of $\operatorname{Rev}[R]$ as

$$
\begin{aligned}
& \geq\left(\frac{q^{\ddagger}(R)}{q_{m}(R)}+0.7 w(R)-\frac{\left.0.7 \log \left(q_{m}(R)\right)\right)}{1-q_{m}(R)}\right) \cdot \frac{2 q_{m}(R)-q^{\ddagger}(R) \cdot(1+1 / 0.7)}{1+v^{*}(R) \cdot\left(1-q_{m}(R)\right)} \\
& \quad+\int_{q_{m}(R)}^{1} p(v(q, R), R) d q .
\end{aligned}
$$

Note that this lower bound is weakly decreasing in $v^{*}(R)$ while holding everything else fixed. Let $v^{*}\left(q_{m}(R), q^{\ddagger}(R), w(R)\right)$ be the upper bound of $v^{*}(R)$ established in Lemma 5.11, Let $b\left(q, q_{m}(R)\right)$ be the lower bound of the optimal bid for an agent with value $v(q, R)$ and revenue curve $R$ established in Lemma 5.12. Then, we can further rewrite the lower bound as

$$
\begin{aligned}
& \geq\left(\frac{q^{\ddagger}(R)}{q_{m}(R)}+0.7 w(R)-\frac{\left.0.7 \log \left(q_{m}(R)\right)\right)}{1-q_{m}(R)}\right) \cdot \frac{2 q_{m}(R)-q^{\ddagger}(R) \cdot(1+1 / 0.7)}{1+v^{*}\left(q_{m}(R), q^{\ddagger}(R), w(R)\right) \cdot\left(1-q_{m}(R)\right)} \\
& \quad+\int_{q_{m}(R)}^{1} \frac{0.7 \log \left(b\left(q, q_{m}(R)\right) \cdot\left(1-q_{m}(R)\right)+1\right)}{1-q_{m}(R)} d q .
\end{aligned}
$$

where the bid $b\left(q, q_{m}(R)\right)$ in the last term can be lower-bounded using Lemma 5.9 .

Therefore, we lower-bound $\boldsymbol{R e v}_{R}(\mathrm{SB})$ as the function of $q_{m}(R), q^{\ddagger}(R), w(R)$. By numerically enumerating all possible parameters, we conclude that $\operatorname{Rev}_{R}(\mathrm{SB}) \geq 0.545$ in this case.

Case (iii) $v^{*}(R) \geq v_{m}(R) / 0.7$ : Lemma 5.11 upper-bounds $v^{*}(R)$ as the function of $q_{m}(R), q^{\ddagger}(R)$ and $w(R)$. By numerically enumerating all possible parameters, we conclude that $v^{*}(R) \geq v_{m}(R) / 0.7$ is not possible for any revenue curve $R$ with $q_{m}(R) \leq 0.62$.

\section{Prior-independent Approximation Lower Bound}

In this section, we show that no mechanism can achieve prior-independent approximation better than 1.07 even when the class of distributions are uniform distributions. Note that point mass distributions are special cases of the uniform distributions. The lower bound we will prove in this 
section holds for more general families of mechanisms than the single-round mechanisms that we introduced in Section 2, Here we will show that even when the agent and the seller have multiple rounds of communication in general messages spaces, no mechanism can achieve prior-independent approximation better than 1.07. However, since our analysis does not hinge on the exact format of the mechanism, we will not formally introduce the model for multi-rounds of communication.

Theorem 6.1. For a single item, a single uniformly distributed agent, and a single valuation sample, the prior-independent approximation ratio for revenue maximization is at least 1.07 .

The main idea for proving Theorem 6.1 is as follows. Consider two scenarios where the valuation distribution of the agent is either uniform between $[1,2]$ or a pointmass with some value $v \in[1,2]$. Note that the optimal mechanism for an agent with value from the uniform distribution $U[1,2]$ is to always allocate the item with expected payment 1 . Thus if the mechanism is optimal for this setting, when the valuation distribution for the agent is actually a pointmass with some value $v \in[1,2]$, the agent can always imitate the type in a uniform distribution $U[1,2]$ to win the item and pay at most 1 in expectation. This indicates that the optimal prior-independent approximation ratio is strictly above 1 . By leveraging the approximation ratio in those two cases, we show that the optimal ratio is at least 1.07 .

Before the proof of Theorem 6.1, we first introduce several notations and present several properties for non-truthful mechanisms $\mathcal{M}$ with prior-independent approximation ratio $\beta$.

Lemma 6.2. For single item, single agent, any distribution $F$ with support $[\underline{v}, \bar{v}]$, for non-truthful mechanism with prior-independent approximation ratio $\beta$, the interim allocation for agent with highest value $\bar{v}$ is $x(\bar{v}, F) \geq \frac{1}{\beta}$.

Proof. Suppose the interim allocation for agent with value $\bar{v}$ is $x(\bar{v}, F)<\frac{1}{\beta}$. Since the interim allocation is monotone, the maximum expected virtual welfare for mechanism under distribution $F$ is less than $1 / \beta$ of the optimal expected virtual welfare, which implies the revenue is less than $1 / \beta$ of the optimal revenue and the approximation ratio for distribution $F$ is higher than $\beta$, a contradiction.

Lemma 6.3. For single item, single agent, and any uniform distribution $F$ with support $[\underline{v}, \bar{v}]$ such that $2 \underline{v} \geq \bar{v}$, for a non-truthful mechanism with prior-independent approximation ratio $\beta$, the interim utility for agent with highest value $\bar{v}$ is $u(\bar{v}, F) \geq \frac{1}{2}\left(\bar{v}-\sqrt{\bar{v}^{2}-\frac{4 \underline{v}}{\beta}}(\bar{v}-\underline{v})\right)$.

Proof. For uniform distribution $F$ with support $[\underline{v}, \bar{v}]$ such that $2 \underline{v} \geq \bar{v}$, the optimal mechanism $\mathrm{OPT}_{F}$ is to post price $\underline{v}$ with expected revenue $\underline{v}$. Suppose the utility for agent with value $\bar{v}$ is $u(\bar{v}, F)<\frac{1}{2}\left(\bar{v}-\sqrt{\bar{v}^{2}-\frac{4 \underline{v}}{\beta}}(\bar{v}-\underline{v})\right)$, the optimal mechanism subject to this constraint is to post price $\bar{v}-u(\bar{v}, F)$, with expected revenue $\frac{u(\bar{v}, F)}{\bar{v}-\underline{v}} \cdot(\bar{v}-u(\bar{v}, F))<\frac{\underline{v}}{\bar{\beta}}$, a contradiction.

Lemma 6.4. For single item, single agent, any point mass distribution $F$ with support $\bar{v}$, for nontruthful mechanism with prior-independent approximation ratio $\beta$, the interim utility for agent with value $\bar{v}$ is $u(\bar{v}, F) \leq \bar{v}(1-1 / \beta)$.

Proof. Suppose the interim utility in this case is $u(\bar{v}, F)>\bar{v}(1-1 / \beta)$, the expected revenue is at most the social welfare minus the expected utility, which is at most $\bar{v}-u(\bar{v}, F)<\frac{\bar{v}}{\beta}$, contradicting the fact that mechanism $\mathcal{M}$ achieves prior-independent approximation ratio $\beta$. 
Proof of Theorem 6.1. Suppose mechanism $\mathcal{M}$ inducing interim allocation and payment rule $x$ and $p$ achieves prior-independent approximation ratio $\beta$. Consider uniform distribution $F$ with support $[1,2]$. By Lemma 6.2 and 6.3, we have $x(2, F) \geq \frac{1}{\beta}$, and $\left.u(2, F) \geq 1-\sqrt{1-1 / \beta}\right)$. For any sample $s \in[1,2]$, the expected allocation and payment of agent with value 2 given the sample $s$ satisfies the constraint that

$$
s \cdot x(2, F, s)-p(2, F, s) \leq s\left(1-\frac{1}{\beta}\right)
$$

otherwise for distribution $F_{s}$ with point mass on $s$, an agent with value $s$ can imitate the behavior of an agent with value 2 in uniform distribution to achieve utility strictly higher than $s(1-1 / \beta)$, and by Lemma 6.4, this contradicts to the assumption that mechanism $\mathcal{M}$ achieves prior-independent approximation ratio $\beta$. Taking expectation over sample $s$ for the left hand side of equation (3), we have

$$
\mathbf{E}_{s}[s \cdot x(2, F, s)-p(2, F, s)] \geq \mathbf{E}_{s}[s \cdot x(2, F, s)]-(2-u(2, F)) \geq \int_{1}^{1+1 / \beta} s d s-(2-u(2, F))
$$

where the last inequality holds because $x(2, F) \geq \frac{1}{\beta}$ and the worst case happens when $x(2, F, s)=0$ for any sample $s \geq 1+1 / \beta$. Taking expectation over sample $s$ for the right hand side of equation (3)), we have

$$
\mathbf{E}_{s}\left[s\left(1-\frac{1}{\beta}\right)\right]=\frac{3}{2}\left(1-\frac{1}{\beta}\right) .
$$

Combining the inequalities, we have

$$
\frac{1}{2}\left(1+\frac{1}{\beta}\right)^{2}-\frac{1}{2}-(1+\sqrt{1-1 / \beta}) \leq \frac{3}{2}\left(1-\frac{1}{\beta}\right) .
$$

By solving the inequality, we have $\beta \geq 1.0737$.

\section{Revelation Gap}

Feng and Hartline (2018) proposed the revelation gap to quantify the difference between the worst case performance of the optimal truthful mechanism and the optimal non-truthful mechanism in prior-independent mechanism design. They showed that a non-trivial revelation gap exists for the welfare maximization problem for agents with budgets. In this section, we show that a revelation gap also exists for the revenue maximization problem when considering the single-item single-agent setting with single-sample access.

Let $\mathbb{M}_{r}$ be the family of truthful mechanisms, the family of mechanisms such that the agent maximizes her utility by truthfully revealing her valuation to the seller, i.e., $b^{*}(v, F)=v$ for all value $v$ and valuation distributions $F$. Let $\mathbb{M}$ be the family of all mechanisms. We define

$$
\beta(\mathrm{MECHS}, \mathrm{DISTS}) \triangleq \min _{\mathcal{M} \in \mathrm{MECHS}} \Gamma(\mathcal{M}, \mathrm{DISTS})
$$

as the optimal prior-independent approximation ratio among the family of mechanisms MECHS. The revelation gap for a family of distributions DISTS is then defined as the ratio $\frac{\beta\left(\mathbb{M}_{r}, \mathrm{DISTS}\right)}{\beta(\mathbb{M}, \mathrm{DISTS})}$.

Definition 7.1. A mechanism is scale-invariant if the interim allocation is invariant of the scale, i.e., $x(\alpha v, \alpha F)=x(v, F)$ for any distribution $F$, valuation $v$ and any $\alpha>0$. 
Allouah and Besbes (2019) characterized the prior-independent approximation ratio of the truthful mechanisms under the assumption of scale-invariance for sample-based pricing mechanisms. Note that in contrast, our lower bound result shown in Theorem 6.1 does not require the assumption on scale-invariance. Here is the formal definition of sample-based pricing mechanisms.

Definition 7.2. Given function $\alpha: \mathbb{R} \rightarrow \Delta(\mathbb{R})$ mapping from the sample to the randomized price, for sample $s$, the sample-based pricing mechanism solicits a non-negative bid $b \geq 0$, allocates the item to the agent if $b \geq \alpha(s)$, and charges the agent $\alpha(s) \cdot \mathbb{1}\{b \geq \alpha(s)\}$.

It can be observed that the bid allocation rules of both sample-bid mechanism and sample-based pricing are similar (i.e. competing against the sample), and the difference is the payment semantics.

Theorem 7.1 (Allouah and Besbes, 2019). Under the assumption of scale-invariance, for singleitem setting with regular valuation distribution, when seller has access to a single sample, the prior-independent approximation ratio of the optimal sample-based pricing mechanism is bounded in $[1.957,1.996]$. Moreover, when the valuation distribution is MHR, the prior-independent approximation ratio is bounded in [1.543, 1.575].

Given an arbitrary valuation distribution and any mechanism that is incentive compatible only for the given valuation distribution, the mechanism may not be equivalent to any sample-based pricing mechanism. The is because the agent only maximizes her utility by taking expectation over the sample. However, we can show that if the mechanism is incentive compatible for all possible prior distributions, then it is equivalent to consider posting a randomized price to the agent based on the realization of the sample, i.e., a sample-based pricing mechanism.

Lemma 7.2. For any mechanism with allocation $\tilde{x}$ and payment $\tilde{p}$ that is incentive compatible and individual rational for all valuation distributions, there exists a sample-based pricing mechanism that generates the same expected allocation and payment pointwise for any valuation of the agent and any realization of the sample.

Proof. First we claim that, for any truthful mechanism with allocation $\tilde{x}$ and payment $\tilde{p}$, the induced allocation rule $\tilde{x}(\cdot, s)$ and payment rule $\tilde{p}(\cdot, s)$ are incentive compatible and individual rational given any realization of the sample $s$.

First we prove the incentive compatibility. Suppose by contradiction, there exists constant $\epsilon>0$, sample $s$ and value $v, v^{\prime}$ such that

$$
v \tilde{x}\left(v^{\prime}, s\right)-\tilde{p}\left(v^{\prime}, s\right) \geq v \tilde{x}(v, s)-\tilde{p}(v, s)+\epsilon .
$$

Let $F$ be an arbitrary distribution with positive density everywhere on the support $[0, \infty)$. Define $H \triangleq u(v, v, F)-u\left(v, v^{\prime}, F\right)$ as the utility loss for value $v$ to misreport $v^{\prime}$ when the distribution is $F$. Given constant $\delta>0$, let $F^{\prime}$ be the distribution such that with probability $1-\delta$, the value of the agent is $s$ and with probability $\delta$, the value is drawn from distribution $F$. It is easy to verify that both $v$ and $v^{\prime}$ are in the support of distribution $F^{\prime}$. Moreover, the utility loss for misreporting $v^{\prime}$ is

$$
u\left(v, v, F^{\prime}\right)-u\left(v, v^{\prime}, F^{\prime}\right) \geq(1-\delta) \epsilon+\delta H
$$

where $(1-\delta) \epsilon+\delta H>0$ for sufficiently small $\delta$. This implies that the mechanism is not incentive compatible for distribution $F^{\prime}$, a contradiction.

Similarly, for individual rationality, if there exists constant $\epsilon>0$, sample $s$ and value $v, v^{\prime}$ such that

$$
v \tilde{x}(v, s)-\tilde{p}(v, s) \leq-\epsilon
$$


there exists a distribution $F^{\prime}$ supported on $[0, \infty)$ such that agent with value $v$ is not individual rational given distribution $F^{\prime}$.

Finally, since for any sample $s$, the induced mechanism is incentive compatible, the allocation $\tilde{x}(v, s)$ is monotone in $v$ for any sample $s$. Moreover, individual rationality implies that the payment of the agent is 0 if she does not win the item. Thus the mechanism can be implemented as sample-based pricing mechanism for any realized sample.

Lemma 7.2 suggest that under the assumption of scale invariance, the bounds on prior-independent approximation ratio of sample-based pricing in Theorem 7.1 carry over to truthful mechanisms. Then combining it with Theorem 5.1 and 6.1, we have the following corollary characterizing the revelation gap under the assumption of scale-invariance.

Corollary 7.3. Under the assumption of scale-invariance, for single-item setting with regular valuation distribution, when seller has access to a single sample, the revelation gap is bounded in [1.066, 1.859]. Moreover, when the valuation distribution is MHR, the revelation gap is bounded in $[1.190,1.467]$. 


\section{References}

Saeed Alaei, Hu Fu, Nima Haghpanah, and Jason Hartline. The simple economics of approximately optimal auctions. In Proc. 54th IEEE Symp. on Foundations of Computer Science, pages 628637. IEEE, 2013.

Saeed Alaei, Jason Hartline, Rad Niazadeh, Emmanouil Pountourakis, and Yang Yuan. Optimal auctions vs. anonymous pricing. Games and Economic Behavior, 118:494-510, 2019.

Amine Allouah and Omar Besbes. Prior-independent optimal auctions. In Proceedings of the 2018 ACM Conference on Economics and Computation, pages 503-503. ACM, 2018.

Amine Allouah and Omar Besbes. Sample-based optimal pricing. In Proceedings of the 2019 ACM Conference on Economics and Computation, pages 391-391. ACM, 2019.

Pablo Azar, Jing Chen, and Silvio Micali. Crowdsourced bayesian auctions. In Proceedings of the 3rd Innovations in Theoretical Computer Science Conference, pages 236-248, 2012.

Pablo D Azar, Robert Kleinberg, and S Matthew Weinberg. Prophet inequalities with limited information. In Proceedings of the twenty-fifth annual ACM-SIAM symposium on Discrete algorithms, pages 1358-1377. SIAM, 2014.

Moshe Babaioff, Yannai A Gonczarowski, Yishay Mansour, and Shay Moran. Are two (samples) really better than one? In Proceedings of the 2018 ACM Conference on Economics and Computation, pages 175-175, 2018.

Richard E Barlow and Albert W Marshall. Tables of bounds for distributions with monotone hazard rate. Journal of the American Statistical Association, 60(311):872-890, 1965.

Gordon M Becker, Morris H DeGroot, and Jacob Marschak. Measuring utility by a single-response sequential method. Behavioral science, 9(3):226-232, 1964.

Jeremy Bulow and Paul Klemperer. Auctions versus negotiations. The American Economic Review, 86(1):180-194, 1996.

Jeremy Bulow and John Roberts. The simple economics of optimal auctions. The Journal of Political Economy, 97:1060-90, 1989.

Bernard Caillaud and Jacques Robert. Implementation of the revenue-maximizing auction by an ignorant seller. Review of Economic Design, 9(2):127-143, 2005.

Shuchi Chawla and Jason Hartline. Auctions with unique equilbria. In Proc. 14th ACM Conf. on Electronic Commerce, pages 181-196, 2013.

Shuchi Chawla, Jason D Hartline, David Malec, and Balasubramanian Sivan. Prior-independent mechanisms for scheduling. In Proceedings of the forty-fifth annual ACM Symposium on Theory of Computing, pages 51-60. ACM, 2013.

Shuchi Chawla, $\mathrm{Hu} \mathrm{Fu}$, and Anna Karlin. Approximate revenue maximization in interdependent value settings. In Proceedings of the fifteenth ACM conference on Economics and computation, pages 277-294. ACM, 2014a. 
Shuchi Chawla, Jason Hartline, and Denis Nekipelov. Mechanism design for data science. In Proceedings of the fifteenth ACM conference on Economics and computation, pages 711-712. ACM, 2014b.

Shuchi Chawla, Jason Hartline, and Denis Nekipelov. A/b testing of auctions. In Proceedings of the 2016 ACM Conference on Economics and Computation, pages 19-20. ACM, 2016.

George Christodoulou, Alkmini Sgouritsa, and Bo Tang. On the efficiency of all-pay mechanisms. In Algorithms-ESA 2015, pages 349-360. Springer, 2015.

Richard Cole and Tim Roughgarden. The sample complexity of revenue maximization. In Proceedings of the forty-sixth annual ACM Symposium on Theory of Computing, pages 243-252, 2014.

José Correa, Paul Dütting, Felix Fischer, and Kevin Schewior. Prophet inequalities for iid random variables from an unknown distribution. In Proceedings of the 2019 ACM Conference on Economics and Computation, pages 3-17, 2019.

José R Correa, Andrés Cristi, Boris Epstein, and José A Soto. The two-sided game of googol and sample-based prophet inequalities. In Proceedings of the Fourteenth Annual ACM-SIAM Symposium on Discrete Algorithms, pages 2066-2081. SIAM, 2020.

Partha Dasgupta and Eric Maskin. Efficient auctions. The Quarterly Journal of Economics, 115 (2):341-388, 2000.

Constantinos Daskalakis and Manolis Zampetakis. More revenue from two samples via factor revealing sdps. In Proceedings of the 21st ACM Conference on Economics and Computation, pages $257-272,2020$.

Nikhil R Devanur, Jason D Hartline, Anna R Karlin, and C Thach Nguyen. Prior-independent multi-parameter mechanism design. In WINE, pages 122-133. Springer, 2011.

Nikhil R Devanur, Jason D Hartline, and Qiqi Yan. Envy freedom and prior-free mechanism design. Journal of Economic Theory, 156:103-143, 2015.

Peerapong Dhangwatnotai, Tim Roughgarden, and Qiqi Yan. Revenue maximization with a single sample. Games and Economic Behavior, 91:318-333, 2015.

Paul Dütting and Thomas Kesselheim. Algorithms against anarchy: Understanding non-truthful mechanisms. In Proceedings of the Sixteenth ACM Conference on Economics and Computation, pages 239-255. ACM, 2015.

Paul Dütting, Federico Fusco, Philip Lazos, Stefano Leonardi, and Rebecca Reiffenhäuser. Efficient two-sided markets with limited information. arXiv preprint arXiv:2003.07503, 2020.

Yiding Feng and Jason D Hartline. An end-to-end argument in mechanism design (priorindependent auctions for budgeted agents). In 2018 IEEE 59th Annual Symposium on Foundations of Computer Science (FOCS), pages 404-415. IEEE, 2018.

Yiding Feng, Jason D Hartline, and Yingkai Li. Optimal auctions vs. anonymous pricing: Beyond linear utility. In Proceedings of the 2019 ACM Conference on Economics and Computation, pages 885-886. ACM, 2019. 
$\mathrm{Hu} \mathrm{Fu}$, Jason Hartline, and Darrell Hoy. Prior-independent auctions for risk-averse agents. In Proceedings of the fourteenth ACM conference on Electronic commerce, pages 471-488, 2013.

$\mathrm{Hu} \mathrm{Fu}$, Nicole Immorlica, Brendan Lucier, and Philipp Strack. Randomization beats second price as a prior-independent auction. In Proceedings of the Sixteenth ACM Conference on Economics and Computation, pages 323-323. ACM, 2015.

Kira Goldner and Anna R Karlin. A prior-independent revenue-maximizing auction for multiple additive bidders. In International Conference on Web and Internet Economics, pages 160-173. Springer, 2016.

Yannai A Gonczarowski and S Matthew Weinberg. The sample complexity of up-to- $\varepsilon$ multidimensional revenue maximization. In 2018 IEEE 59th Annual Symposium on Foundations of Computer Science (FOCS), pages 416-426. IEEE, 2018.

Chenghao Guo, Zhiyi Huang, and Xinzhi Zhang. Settling the sample complexity of single-parameter revenue maximization. In Proceedings of the 51st Annual ACM Symposium on Theory of Computing, pages 662-673, 2019.

Jason Hartline and Samuel Taggart. Sample complexity for non-truthful mechanisms. In Proc. 20th ACM Conf. on Economics and Computation, pages 399-416, 2019.

Jason Hartline, Darrell Hoy, and Sam Taggart. Price of anarchy for auction revenue. In Proceedings of the fifteenth ACM conference on Economics and computation, pages 693-710, 2014.

Jason Hartline, Aleck Johnsen, and Yingkai Li. Benchmark design and prior-independent optimization. In 61st Annual Symposium on Foundations of Computer Science, to appear, 2020.

Jason D Hartline. Mechanism design and approximation. 2016.

Darrell Hoy, Samuel Taggart, and Zihe Wang. A tighter welfare guarantee for first-price auctions. In Proceedings of the 50th Annual ACM Symposium on Theory of Computing, pages 132-137, 2018.

Zhiyi Huang, Yishay Mansour, and Tim Roughgarden. Making the most of your samples. SIAM Journal on Computing, 47(3):651-674, 2018.

Matthew O Jackson. A crash course in implementation theory. Social choice and welfare, 18(4): 655-708, 2001.

Yaonan Jin, Pinyan Lu, Qi Qi, Zhihao Gavin Tang, and Tao Xiao. Tight approximation ratio of anonymous pricing. In Proceedings of the 51st Annual ACM Symposium on Theory of Computing, pages $674-685,2019$.

Yaonan Jin, Pinyan Lu, Zhihao Gavin Tang, and Tao Xiao. Tight revenue gaps among simple mechanisms. SIAM Journal on Computing, 49(5):927-958, 2020.

Eric Maskin. Auctions, development, and privatization: Efficient auctions with liquidityconstrained buyers. European Economic Review, 44(4-6):667-681, 2000.

Jamie H Morgenstern and Tim Roughgarden. On the pseudo-dimension of nearly optimal auctions. In Advances in Neural Information Processing Systems, pages 136-144, 2015.

R. Myerson. Optimal auction design. Mathematics of Operations Research, 6:58-73, 1981. 
Tim Roughgarden, Inbal Talgam-Cohen, and Qiqi Yan. Supply-limiting mechanisms. In Proceedings of the 13th ACM Conference on Electronic Commerce, pages 844-861. ACM, 2012.

Tim Roughgarden, Vasilis Syrgkanis, and Eva Tardos. The price of anarchy in auctions. Journal of Artificial Intelligence Research, 59:59-101, 2017.

Tim Roughgarden, Inbal Talgam-Cohen, and Qiqi Yan. Robust auctions for revenue via enhanced competition. Operations Research, 68(4):1074-1094, 2020.

Jerome H Saltzer, David P Reed, and David D Clark. End-to-end arguments in system design. ACM Transactions on Computer Systems (TOCS), 2(4):277-288, 1984.

Vasilis Syrgkanis and Eva Tardos. Composable and efficient mechanisms. In Proceedings of the forty-fifth annual ACM Symposium on Theory of Computing, pages 211-220, 2013. 


\section{A Numerical Analysis}

In Section 5, we bound the prior-independent approximation ratio of the sample-bid mechanism by enumerating the possible choices of given parameters. One concern is that the parameters are selected from a continuous interval, and the revenue for valuation distributions with parameters that are not evaluated on discretized points may be far from the revenue on discretized points. In this section, we formally show that this is not the case for our analysis. To provide a theoretical lower bound on all possible distributions, we will present a unified lower bound on the revenue for distributions with parameters between discretized points. We will formalize this approach for the numerical calculation for Lemma 5.7, and the numerical calculation for other lemmas and theorems hold similarly.

By the proof of Lemma 5.7, for any revenue curve $R$ in Figure 3e parameterized by monopoly quantile $q_{m} \in\left[\underline{q}_{m}, \bar{q}_{m}\right]$ and revenue $r_{0} \in\left[\underline{r}_{0}, \bar{r}_{0}\right]$ for quantile 0 , the revenue of the seller is lower bounded by $p\left(v^{*}(R), R\right) \cdot q^{*}(R)$ where $v^{*}(R)$ is the critical value with bid above monopoly price and $q^{*}(R)$ is the quantile for critical value. Note that it is sufficient for us to consider revenue curves $R$ such that $v^{*}(R)$ is at least the monopoly price. Next we show how to provide bounds on parameters $\underline{q}_{m}, \bar{q}_{m}, \underline{r}_{0}, \bar{r}_{0}$, as well as lower bounds on $p\left(v^{*}(R), R\right)$ and $q^{*}(R)$ using parameters $\underline{q}_{m}, \bar{q}_{m}, \underline{r}_{0}, \bar{r}_{0}$.

Lemma A.1. There exists efficiently computed set $S \subseteq \mathbb{R}^{4}$ and function $\tau: \mathbb{R}^{4} \rightarrow \mathbb{R}$ such that for any revenue curve $R$ in Figure 3 e parameterized by monopoly quantile $q_{m} \in\left[\underline{q}_{m}, \bar{q}_{m}\right]$ and revenue $r_{0} \in\left[\underline{r}_{0}, \bar{r}_{0}\right]$ for quantile 0 , we have

$$
\begin{aligned}
& \text { 1. } v^{*}(R) \geq v_{m}(R) \text { only if }\left(\underline{q}_{m}, \bar{q}_{m}, \underline{r}_{0}, \bar{r}_{0}\right) \in S \text {; } \\
& \text { 2. } p\left(v^{*}(R), R\right) \cdot q^{*}(R) \geq \tau\left(\underline{q} \underline{q}_{m}, \bar{q}_{m}, \bar{r}_{0}\right) \text { if }\left(\underline{q}_{m}, \bar{q}_{m}, \underline{r}_{0}, \bar{r}_{0}\right) \in S \text {. }
\end{aligned}
$$

Proof. First we illustrate how to find the desirable set $S$ by numerical calculation. Note that the requirement is such that the critical value for bidding above the monopoly price is above monopoly price, i.e., $v^{*}(R) \geq v_{m}(R)$. By Lemma 5.6, it is sufficient to verify that the optimal utility of value $v_{m}(R)$ for bidding above $v_{m}(R)$ is positive. Note that by Lemma 3.2, the optimal bid above the monopoly price is $b=\frac{v_{m}}{\alpha}+\frac{1-r_{0}}{1-q_{m}}$, with expected utility

$$
u\left(v_{m}, b\right)=\frac{1}{q_{m}} \cdot\left(1-q_{b}\right)-\alpha\left(b \cdot q_{b}+r_{0} \log \left(\frac{q_{m}}{q_{b}}\right)+\frac{\left(1-r_{0}\right)\left(q_{m}-q_{b}\right)}{q_{m}}-\log q_{m}\right)
$$

where $q_{b}=\frac{r_{0}}{b-\frac{1-r_{0}}{q_{m}}}$. Since $q_{m} \in\left[\underline{q}_{m}, \bar{q}_{m}\right]$ and $r_{0} \in\left[\underline{r}_{0}, \bar{r}_{0}\right]$, a sufficient condition for $u\left(v_{m}, b\right)>0$ is that

$$
\frac{1}{\bar{q}_{m}} \cdot\left(1-\bar{q}_{b}\right)-\alpha\left(\bar{b} \cdot \bar{q}_{b}+\bar{r}_{0} \log \left(\frac{\bar{q}_{m}}{\underline{q}_{b}}\right)+\frac{\left(1-\underline{r}_{0}\right)\left(\bar{q}_{m}-\underline{q}_{b}\right)}{\underline{q}_{m}}-\log \underline{q}_{m}\right)>0
$$

where $\bar{b}=\frac{1}{\alpha q_{m}}+\frac{1-\underline{r}_{0}}{1-\bar{q}_{m}}, \bar{q}_{b}=\frac{\alpha \bar{r}_{0} \bar{q}_{m}\left(1-\underline{q}_{m}\right)}{1-\bar{q}_{m}+\alpha\left(1-\bar{r}_{0}\right)}$ and $\underline{q}_{b}=\frac{\alpha \underline{r}_{0} \underline{q}_{m}\left(1-\bar{q}_{m}\right)}{1-\underline{q}_{m}+\alpha\left(1-\underline{r}_{0}\right)}$. Note that the above inequality can be easily verified on discretized points.

Next we construct the function $\tau\left(\underline{q}_{m}, \bar{q}_{m}, \underline{r}_{0}, \bar{r}_{0}\right)$ lower bound the revenue $p\left(v^{*}(R), R\right) \cdot q^{*}(R)$. First note that we can enumerate the value above monopoly price and find the minimum value that the interim utility is strictly positive. That is, given value $v \geq v_{m}$, the optimal bid above the monopoly 
price is $b=\frac{v}{\alpha}+\frac{1-r_{0}}{1-q_{m}}$, with expected utility

$$
\begin{aligned}
u(v, b) & =v \cdot\left(1-q_{b}\right)-\alpha\left(b \cdot q_{b}+r_{0} \log \left(\frac{q_{m}}{q_{b}}\right)+\frac{\left(1-r_{0}\right)\left(q_{m}-q_{b}\right)}{q_{m}}-\log q_{m}\right) \\
& \geq v \cdot\left(1-\bar{q}_{b}\right)-\alpha\left(\bar{b} \cdot \bar{q}_{b}+\bar{r}_{0} \log \left(\frac{\bar{q}_{m}}{\underline{q}_{b}}\right)+\frac{\left(1-\underline{r}_{0}\right)\left(\bar{q}_{m}-\underline{q}_{b}\right)}{\underline{q}_{m}}-\log \underline{q}_{m}\right)>0,
\end{aligned}
$$

where $\bar{b}=\frac{v}{\alpha}+\frac{1-\underline{r}_{0}}{1-\bar{q}_{m}}, \bar{q}_{b}=\frac{\alpha \bar{r}_{0} \bar{q}_{m}\left(1-\underline{q}_{m}\right)}{v \underline{q}_{m}\left(1-\bar{q}_{m}\right)+\alpha\left(1-\bar{r}_{0}\right)}$ and $\underline{q}_{b}=\frac{\alpha \underline{r}_{0} \underline{q}_{m}\left(1-\bar{q}_{m}\right)}{v \bar{q}_{m}\left(1-\underline{q}_{m}\right)+\alpha\left(1-\underline{r}_{0}\right)}$. Let $v^{*}$ be the minimum value that satisfies the above inequality. Then we have $v^{*} \geq v^{*}(R)$, and hence

$$
q^{*}(R) \geq q\left(v^{*}, R\right)=\frac{\alpha r_{0} q_{m}\left(1-q_{m}\right)}{v q_{m}\left(1-q_{m}\right)+\alpha\left(1-r_{0}\right)} \geq \frac{\alpha \underline{r}_{0} \underline{q}_{m}\left(1-\bar{q}_{m}\right)}{v \bar{q}_{m}\left(1-\underline{q}_{m}\right)+\alpha\left(1-\underline{r}_{0}\right)} .
$$

Moreover, we can similar construct an upper bound on the utility $u(v, b)$ and let $\underline{v}^{*}$ be the largest value such that the upper bound on the utility is at most 0 . Thus, we have $v^{*}(R) \geq \underline{v}^{*}$ and hence

$$
\begin{aligned}
p\left(v^{*}(R), R\right) \geq p\left(\underline{v}^{*}, R\right) & =\alpha\left(b \cdot q_{b}+r_{0} \log \left(\frac{q_{m}}{q_{b}}\right)+\frac{\left(1-r_{0}\right)\left(q_{m}-q_{b}\right)}{q_{m}}-\log q_{m}\right) \\
& \geq \alpha\left(\underline{b} \cdot \underline{q}_{b}+\underline{r}_{0} \log \left(\frac{\underline{q}_{m}}{\bar{q}_{b}}\right)+\frac{\left(1-\bar{r}_{0}\right)\left(\underline{q}_{m}-\bar{q}_{b}\right)}{\bar{q}_{m}}-\log \bar{q}_{m}\right)
\end{aligned}
$$

where $\underline{b}=\frac{v^{*}}{\alpha}+\frac{1-\bar{r}_{0}}{1-\underline{q}_{m}}, \bar{q}_{b}=\frac{\alpha \bar{r}_{0} \bar{q}_{m}\left(1-\underline{q}_{m}\right)}{\underline{v}^{*} \underline{q}_{m}\left(1-\bar{q}_{m}\right)+\alpha\left(1-\bar{r}_{0}\right)}$ and $\underline{q}_{b}=\frac{\alpha \underline{r}_{0} \underline{q}_{m}\left(1-\bar{q}_{m}\right)}{\underline{v}^{*} \bar{q}_{m}\left(1-\underline{q}_{m}\right)+\alpha\left(1-\underline{r}_{0}\right)}$. By combining the inequalities, we have an lower bound on $p\left(v^{*}(R), R\right) \cdot q^{*}(R)$ as a function of $\left(q_{m}, \bar{q}_{m}, \underline{r}_{0}, \bar{r}_{0}\right)$. By discretizing the feasible set and enumerating for all discretized points, we have a unified lower bound on revenue for all possible distributions. 